\title{
Nitrogen Assimilation in Bacteria
}

\author{
Antonia Herrero ${ }^{1 *}$, Enrique Flores ${ }^{1}$ and Juan Imperial ${ }^{2,3}$ \\ ${ }^{1}$ Instituto de Bioquímica Vegetal y Fotosíntesis, Consejo Superior de Investigaciones \\ Científicas and Universidad de Sevilla, Américo Vespucio 49, E-41092 Seville, Spain
}

${ }^{2}$ Centro de Biotecnología y Genómica de Plantas, Universidad Politécnica de Madrid Instituto Nacional de Investigación y Tecnología Agraria y Alimentaria, Campus de Montegancedo, Ctra. M-40, km 38, E-28223 Pozuelo de Alarcón, Spain

${ }^{3}$ Present address: Instituto de Ciencias Agrarias, Consejo Superior de Investigaciones Científicas, Serrano 115 bis, E-28006 Madrid, Spain

*Corresponding author. E-mail: herrero@ibvf.csic.es; Tel.: 3495448 9522. Fax: 954460165.

Keywords: Amino acid catabolism; Ammonium uptake; Cyanophycin; GlnB/GInK; Glutamine synthetase; Glutamate synthase; Heterocyst differentiation; Nitrate assimilation; Nitrogen control; Nitrogen fixation; Nitrogenase; NtcA; NtrB/NtrC; Respiratory Nif protection; TnrA; Urea assimilation. 


\section{Abstract}

Nitrogen is one of the most abundant elements in living matter and, hence, sources of nitrogen are macro-nutrients. Most bacteria can assimilate ammonium, which is the inorganic nitrogen compound that is incorporated into organic material. The ammonium transporter AmtB/Amt1 is ubiquitous in bacteria, permitting scavenging ammonium from the extracellular medium. Once inside the cell, ammonium is generally incorporated into carbon skeletons through the very efficient, but energy-consuming, glutamine synthetase-glutamate synthase cycle. This cycle produces glutamine and glutamate, which distribute nitrogen in cellular anabolism. Because ammonium is not always available, bacteria have evolved the assimilation of many different inorganic and organic nitrogen-containing compounds. Sources of nitrogen alternative to ammonium frequently include nitrate and urea as well as atmospheric nitrogen in the process of nitrogen fixation, carried out only by prokaryotes. More complex organic sources of nitrogen can also be assimilated by many bacteria, and they include several amino acids, nitrogenous bases and even some xenobiotics. Pathways for assimilation of nitrogen sources alternative to ammonium are generally expressed only if the bacteria experience nitrogen deprivation. Regulators that control expression of the genes encoding those pathways include molecular systems as different as the two-component system NtrBC of enterobacteria, the MerR-family transcription factors TnrA and GInR of Bacillus subtilis and the CRP-family NtcA transcription factor of cyanobacteria. Nonetheless, the C-to-N balance of the cells is generally sensed as levels of 2-oxoglutarate -which accumulates under nitrogen deprivation-, sometimes accompanied by sensing of glutamine -which accumulates under nitrogen sufficiency. Under high C-to-N balance conditions (i.e., nitrogen deficiency), these regulators activate the expression of genes encoding proteins for the assimilation of nitrogen sources alternative to ammonium, but in many cases induction of the genes by the corresponding nitrogen source is necessary as well. 


\section{Introduction}

Nitrogen is a main component of living organisms, accounting for 10 to $15 \%$ of the dry weight of bacteria, which represents $1 / 4$ of their carbon contents. In living matter, nitrogen is present in nucleic acids and proteins, the defining molecules of cells, and in bacteria it is also present in cell walls. Moreover, besides its importance for the organism that conducts the process, biological nitrogen assimilation is a crucial part of the global carbon and nitrogen cycles, and has thus a major impact on climate and planetary composition at global scales.

Nitrogen is found in different molecular forms in all natural environments in our planet, and these forms are interconverted with a massive participation of microorganisms (Fig. 1). In soils, nitrogen is found in organic forms, mainly derived from animal waste and organic matter decomposition, or inorganic compounds, mainly ammonium, although other compounds such as nitrates, nitrites and nitrogenous oxides are also present. The relative composition of nitrogen in land depends very much on the particular environment. In fresh waters the content of nitrogenous compounds is also variable and could be related to that of in-shore lands. In oceans, nitrate, ammonium and urea can reach micromolar concentrations. The main component of the Earth's atmosphere is $\mathrm{N}_{2}(\mathrm{~N} \equiv \mathrm{N}, 78 \%)$, which to a large extent is of biological origin. <Fig. 1 near here>

The bulk of the biological transformations of nitrogenous compounds in land and water environments take place through the processes of: ammonification, carried out by bacteria and fungi and whereby organic nitrogen is transformed into ammonium; bacterial nitrification, which involves the conversion of ammonium into nitrites and nitrates; nitrate and nitrite assimilation, the intracellular conversion by plants and microorganisms of nitrate and nitrite into ammonium; and the anaerobic processes of bacterial denitrification and anammox, which involves the conversion of nitrate or ammonium, respectively, into $\mathrm{N}_{2}$ gas, which is liberated to the atmosphere (Fig. 1). Although some transformation of atmospheric $\mathrm{N}_{2}$ into nitric oxides and nitrate takes place through natural atmospheric phenomena such as lightning and volcanic 
activity, and into ammonium by industrial processes to produce fertilizers, the main pathway of transformation of $\mathrm{N}_{2}$ nitrogen is through biological $\mathrm{N}_{2}$ fixation. This process, which is exclusively conducted by prokaryotes, either free-living or in symbiosis with plants or algae, represents the main way to convert $\mathrm{N}$ into the biologically utilizable pool (Fig. 1). Thus, it is of primordial importance to maintain the global ecological stasis.

The oxidative processes of nitrification (by nitrifying bacteria) or anammox and the reduction of nitrate by denitrifying bacteria are used for the production of reductant and energy, while the dissimilatory reduction of nitrate and nitrite can be used to generate energy or serve detoxification purposes (e.g., to eliminate excess of reductant or nitrite). In contrast, the assimilatory reduction of nitrate, nitrite and $\mathrm{N}_{2}$ is, together with the direct incorporation of ammonium or of some forms of organic N, directed to building organic matter for growth, often in an energy-dependent manner (Fig. 1). In the following sections, we describe these assimilatory processes (Fig. 2) and their regulatory control. Given their central nature, we first cover ammonium assimilation and its regulation. We then study the assimilation of alternative $\mathrm{N}$ sources and their specific regulatory mechanisms, and conclude emphasizing the global systems of $\mathrm{N}$ control. $\quad$ <Fig. 2 near here>

\section{Ammonium assimilation}

\section{Ammonium uptake}

The ammonium ion $\left(\mathrm{NH}_{4}^{+}\right)$is a key compound in the assimilation of nitrogen in numerous biological systems because it is the inorganic form of nitrogen that is incorporated into carbon skeletons. Ammonium present in the environment can be assimilated by many bacteria, yeast, fungi, algae, and plants. Ammonium solutions always contain ammonia $\left(\mathrm{NH}_{3}\right)\left(p K_{\mathrm{a}}\right.$ [ammonium/ammonia], 9.25), which can diffuse through biological membranes. Diffusion of ammonia followed by trapping of intracellular ammonium by glutamine synthetase can 
represent a significant process for nitrogen acquisition, especially in organisms such as some bacteria that can grow in alkaline media. This process would be less important in fungi that grow in acidic media. However, in numerous organisms there is evidence for the presence of ammonium transport systems that can be conveniently probed with ${ }^{14} \mathrm{C}$-labeled methylammonium (the $\mathrm{p} K_{\mathrm{a}}$ of methylammonium/methylamine is 10.65). Genes encoding ammonium/methylammonium permeases were first identified in Saccharomyces cerevisiae (MEP) and Arabidopsis thaliana (AMT1). Of note, the Rhesus proteins in chordates are homologues of the ammonium transporters. Genes coding for proteins similar to MEP- and AMT1-encoded proteins are ubiquitous in bacterial genomes, where they are denoted amtB or a variation thereof. For instance, a gene that has been denoted amt1 is widespread in cyanobacteria.

\section{The Amt proteins}

Bacterial Amt proteins contain 400 to 500 -amino acid residues. They are integral membrane proteins that normally have 12 transmembrane segments. The $\mathrm{N}$-terminal transmembrane segment is frequently part of a signal sequence that is removed after integration of the protein into the membrane. The crystal structures of the AmtB proteins from Escherichia coli and the euryarchaeon Archaeoglobus fulgidus are available, showing that AmtB forms trimers in which each monomer provides a hydrophobic pore to accommodate and conduct the substrate. Although the nature of the transferred substrate $\left(\mathrm{NH}_{4}{ }^{+}\right.$or $\left.\mathrm{NH}_{3}\right)$ has been debated, recent work supports early conclusions based on physiological studies that the transferred substrate is the ammonium ion, although the mechanism of transport could involve a deprotonation step and parallel transfer of a proton. The net result of transport is concentration of ammonium inside the cells dependent on the membrane potential (negative inside the bacterial cells), and incorporation of ammonium into glutamine catalyzed by glutamine synthetase (GS). This has 
been clearly shown also in studies using $\left[{ }^{14} \mathrm{C}\right]$ methylammonium as a substrate, in which intracellularly accumulated $\left[{ }^{14} \mathrm{C}\right]$ methylammonium and $\left[{ }^{14} \mathrm{C}\right]$ methylglutamine are detected.

Bacterial Amt proteins have a very high affinity for ammonium $\left(K_{\mathrm{s}}\right.$ values below micromolar), and the expression of amt genes is commonly repressed by high concentrations of ammonium in a process that involves the global nitrogen regulatory transcriptional system, e.g., Ntr in the enterobacteria or NtcA in cyanobacteria (see section on Nitrogen control). Indeed, the Amt proteins are not needed for ammonium assimilation when ammonium is provided at high levels (e.g., in the millimolar range at a neutral pH), as demonstrated by the ability of amt knockout mutants to grow using ammonium as a nitrogen source.

\section{Regulation of AmtB}

The amtB gene is frequently clustered together with $g \ln K$, which encodes a $\mathrm{P}_{\mathrm{II}}$-like protein. As described in the section on $\mathrm{N}$ control, $\mathrm{P}_{\|}$proteins are sensors of the nitrogen status of the cell. In enterobacteria, $\mathrm{P}_{\|}$proteins are modified by uridylylation under low-nitrogen conditions. In the presence of a non-limiting ammonium concentration, the GlnK trimer is deuridylylated and binds the AmtB trimer, inhibiting ammonium transport (Fig. 3). <Fig. 3 near here>

In conclusion, the Amt proteins function to scavenge ammonium when this substrate is found at low extracellular concentrations. Because of the paired presence of ammonium and diffusible ammonia in solution (respective concentrations depending on $\mathrm{pH}$ ), when the concentration of the $\mathrm{NH}_{4}^{+} / \mathrm{NH}_{3}$ pair is too high, the cells respond repressing amt gene expression and frequently also blocking AmtB activity. These responses could impede the operation of a futile cycle in which the ammonium concentrated within the cells leaks out due to unavoidable ammonia diffusion.

\section{Incorporation of intracellular ammonium into carbon skeletons}


We will show throughout this chapter that all $\mathrm{N}$ sources eventually yield intracellular ammonium. This is not surprising, since $\mathrm{N}_{\text {in }} \mathrm{NH}_{4}{ }^{+}$is at the same oxidation state as the organic $\mathrm{N}$ present in biological molecules. Intracellular ammonium, either coming from $\mathrm{NH}_{4}{ }^{+}$uptake or from the assimilation of alternative $\mathrm{N}$ sources, is incorporated into $\mathrm{C}$ skeletons by reaction with organic acids to yield amino acids. 2-oxoglutarate is the preferred ammonium acceptor, to yield glutamate and glutamine. Glutamate and glutamine provide the $\mathrm{N}$ for all the other amino acids, while glutamine provides $\mathrm{N}$ for nucleotide and amino sugar biosynthesis.

\section{Enzymatic routes}

Glutamate and glutamine are the main entry points of $\mathrm{N}$ to the organic pool in bacteria. Glutamine synthetases (GS) catalyze the amidation of glutamate to yield glutamine at the expense of one molecule of ATP. A second enzyme, glutamate synthase (GOGAT), produces two molecules of glutamate from one of 2-oxoglutarate and one of glutamine, consuming two reducing equivalents. Through the concerted action of the GS/GOGAT two-enzyme system (Fig. 2), ammonium is incorporated into 2-oxoglutarate with high affinity, but one ATP is consumed per glutamate formed. A third enzyme, glutamate dehydrogenase (GDH) can catalyze the reductive amination of 2-oxoglutarate to yield glutamate using a reduced pyridine nucleotide, but the reaction equilibrium is heavily displaced in the opposite direction, and GDHs are usually catabolic. In enterobacteria and in many other bacteria, both systems coexist. Other bacteria can lack GDH or, more rarely, GOGAT. In enterobacteria, GS is a dodecamer of identical subunits, each with an active site. In other bacteria, GS can be much simpler. Still other bacteria contain more than one type of GS, which can increase the flexibility of their $\mathrm{N}$ assimilation pathways. In enterobacteria, GOGAT is a heterodimeric, iron-sulfur flavoprotein that uses NADPH. In other bacteria, NADH or even ferredoxin are preferred over NADPH, and it is not uncommon for bacteria to harbor more than one type of GOGAT. 
Other, minor, ways of ammonium incorporation into organic skeletons exist, such as $\mathrm{NAD}^{+}$synthase or the amidation of aspartate catalyzed by asparagine synthetase, but one of them in particular, the synthesis of alanine from pyruvate and ammonium, catalyzed by alanine dehydrogenase, appears to be important in diazotrophic legume bacteroids of some rhizobia, both as a sink of fixed ammonium and as a $\mathrm{N}$-delivery molecule to the plant.

\section{Regulation}

In many bacteria, the $g \ln A$ gene is regulated by the availability of ammonium through the global $\mathrm{N}$-control circuits. Additionally, in E. coli, the GS activity is regulated by a tight bicyclic regulatory cascade (Fig. 4), which shares components of N-regulation. In this circuit, each of the $12 \mathrm{GS}$ subunits can be modified through adenylylation/deadenylylation by GInE, a bifunctional adenylyl transferase/removing enzyme (ATase). Adenylylation reduces the catalytic activity of GS, and although high glutamine levels do not directly inhibit GS activity, they determine ATasemediated adenylylation. Conversely, high levels of 2-oxoglutarate stimulate deadenylylation. In turn, ATase activity is under control of the $\mathrm{P}_{\| /} /$uridylyl transferase (UTase, the $g \ln D$ gene product) cascade: GInB (and to a lower extent GInK) stimulates adenylylation and GInB-UMP (GlnK-UMP) stimulates deadenylylation. ATase appears to preferentially sense two forms of the $P_{\|}$protein: trimers bound to one 2-oxoglutarate stimulate adenylylation, while fully uridylylated and 2oxoglutarate saturated trimers stimulate deadenylylation.

<Fig. 4 near here>

\section{Assimilation of organic sources of nitrogen}

A large diversity of nitrogenous organic compounds can be used as sources of nitrogen in the bacterial world. Relatively simple compounds that can be used by different bacteria include urea, some amino acids, amino sugars and simple amine-containing compounds such as methylamine, ethanolamine or some polyamines. Utilization of amino acids sometimes implies 
previous degradation of peptides that should be considered the actual nitrogen source. Other complex compounds such as heterocyclic nitrogenous compounds including, e.g., nitrogenous bases (pyrimidines and purines) or xenobiotics such as atrazine can also be used by some bacteria. The utilization of an organic nitrogenous compound as a nitrogen source requires the uptake of the substrate into the bacterial cell. Then, specific metabolic route(s) catabolize the substrate to produce ammonium that is incorporated into amino acids. In many cases, the bacteria completely mineralize the substrate releasing ammonium. Some amino acids, on the other hand, are catabolized producing glutamate, which is the major distributor of nitrogen in metabolism; however, degradation of the amino acid should also produce ammonium to be used by GS to synthetize glutamine (Fig. 2).

The myriad of bacterial pathways involved in the utilization of organic sources of nitrogen cannot be covered here. We will therefore outline examples of the pathways of utilization of a few substrates that can enlighten the reader about the variety of catabolic pathways displayed in the bacterial world.

\section{Urea}

Urea $\left(\mathrm{CO}\left[\mathrm{NH}_{2}\right]_{2}\right)$ is a simple organic compound present in both ocean and fresh waters as well as in the soil (in part because of its use as fertilizer), frequently at micromolar concentrations or below, and it can be assimilated by bacteria belonging to different phylogenetic groups. Although urea at relatively high (millimolar) concentrations can permeate biological membranes permitting at least slow growth, it can also be incorporated into many bacteria by an active transporter. Once inside the cell, urea is degraded by urease to produce $\mathrm{CO}_{2}$ and ammonium, which is incorporated into organic material (Fig. 2).

\section{Genes and proteins}


Active uptake of urea appears essential to assimilate this compound when found below micromolar concentration. Bacterial urea uptake is mediated by an ABC-type transporter that was first identified in cyanobacteria. $A B C$ uptake transporters typically comprise one periplasmic solute-binding protein, two integral membrane (transmembrane or permease) proteins/domains and two nucleotide-binding proteins/domains that hydrolyze ATP in the cytoplasm. The urea transporter is encoded by five genes that form an operon with a typical structure for $A B C$ transporter-encoding operons, including $\left(5^{\prime}\right.$ to $\left.3^{\prime}\right)$ the genes for the periplasmic substrate-binding protein (UrtA), the transmembrane proteins (UrtB, UrtC), and the nucleotide-binding proteins (UrtD, UrtE) (Fig. 5). This transporter shows a high affinity for urea ( $K_{\mathrm{s}}$ about $0.1 \mu \mathrm{M}$ in the cyanobacterium Anabaena sp. PCC 7120), keeping the concentration of urea in the cytoplasm to a level usable by urease.

<Fig. 5 near here>

Urease (urea amidohydrolase) catalyzes the hydrolysis of urea to produce ammonia and carbamate, which spontaneously decomposes into another molecule of ammonia and carbonic acid. Urease is a trimer of a complex formed itself by three subunits encoded by the ureA, ure $B$ and ureC genes. Each UreABC complex bears, located in UreC, a catalytic site that contains two nickel ions bridged by a carbamylated lysine and coordinated by histidine and aspartate residues. Synthesis of active urease is a complex GTP-dependent process that requires the products of accessory genes, ureD, ureE, ureF and ureG, which have a role in metallocenter assembly (Fig. 5).

The urease structural and maturation genes are generally clustered together in bacterial genomes and, interestingly, they are also frequently clustered with the urea $A B C$ transporterencoding genes, which may be found in the same or opposite orientation (Fig. 5). This arrangement may suggest that all these genes are subjected to concerted regulation, but also (or alternatively) that they have been received as a package by horizontal gene transfer in the bacterium where the cluster is found. 


\section{Regulation}

The urt genes encoding the urease transporter are subjected to $\mathrm{N}$ control, so that they are expressed at high levels only under nitrogen deficiency. This is the case in, e.g., the cyanobacterium Anabaena sp. PCC 7120, where the urt operon is transcribed from an NtcAdependent promoter, and Mycobacterium smegmatis, where the urt genes are under the control of the response regulator GInR. In contrast, urease-encoding genes are expressed constitutively in some bacteria but regulated in others. Amongst the latter, the Klebsiella pneumoniae ure genes are transcribed from two promoters, one of which is strictly dependent on the nitrogen assimilation regulator Nac, which is a LysR-type factor that activates $\sigma^{70}$ dependent transcription in the absence of any effector (i.e., Nac is not regulated at the protein activity level). Instead, the expression of the nac gene is dependent on the Ntr system (see $\mathrm{N}$ control, the E. coli cascade system).

In conclusion, the urea transporter appears to have an important role in urea scavenging, permitting the proliferation of urea-assimilating bacteria in natural habitats where urea may be found at low concentrations. A urease regulated in a similar way as the transporter would contribute to this function. On the other hand, in some bacteria urease participates in specific metabolic pathways (e.g., in arginine catabolism; see Fig. 2) that require constitutive expression or expression regulated independently of nitrogen control. Additionally, urease has an important role in virulence and/or adaptation to acidic environments in bacteria such as Streptococcus sp., Proteus sp. and Helicobacter sp., but this topic is outside the scope of this chapter.

\section{Aspartate, asparagine, glutamate and glutamine}

These amino acids can be used as sources of nitrogen by many bacteria. The utilization of glutamate and glutamine is straightforward. Specific glutamate uptake systems of different transporter families are found in bacteria, including secondary $\mathrm{H}^{+}$- or $\mathrm{Na}^{+}$-dependent 
transporters such as the $\mathrm{Na}^{+}$-dependent GltS of $E$. coli, or $\mathrm{ABC}$ transporters such as GluABCD from Corynebacterium glutamicum. There are also transporters for glutamate and related compounds such as the $\mathrm{H}^{+}$-dependent symporters for aspartate or glutamate, TRAP (Tripartite ATP-independent periplasmic) transporters for dicarboxylates, or ABC transporters for aspartate/glutamate/asparagine/glutamine in numerous bacteria. Once inside the cells, glutamate can be used directly in metabolism or deaminated by glutamate dehydrogenase, releasing ammonium. Regarding glutamine, it can be taken up by shared transporters and also by specific transporters such as the $A B C$ transporter GInHPQ of enterobacteria. Glutamine can be deaminated by specific glutaminases (glutamine amidohydrolases) producing glutamate and ammonium, but it can also be used to distribute nitrogen directly in metabolism through amidotransferases that bear glutaminase domains. The utilization of glutamine is however subjected to some nuances in organisms, such as the enterobacteria, in which glutamine is itself an indicator of the carbon-to-nitrogen balance of the cell. Hence, although abundant glutamine should be perceived as nitrogen excess (see $\mathrm{N}$ control, the $E$. coli cascade system), growth of $E$. coli on glutamine as a nitrogen source induces the Ntr response.

Aspartate can be incorporated into bacterial cells by secondary transporters of the Dicarboxylate/Amino Acid:Cation $\left(\mathrm{Na}^{+}\right.$or $\mathrm{H}^{+}$) Symporter (DAACS) Family such as GltP (in E. coli) or GltT (in B. subtilis), or by ABC transporters such as GItIJKL (in E. coli). Once inside the cell, aspartate can be subjected to transamination to 2-oxoglutarate catalyzed by aspartate aminotransferase to produce oxaloacetate and glutamate, or to deamination catalyzed by aspartase (aspartate-ammmonia lyase) to produce fumarate and ammonium. Many bacteria, including phylogenetically distant organisms such as E. coli and B. subtilis, can metabolize aspartate with these two enzymes making nitrogen available for anabolism in two forms: glutamate and ammonium (Fig. 2).

Asparagine can provide nitrogen directly in the form of ammonium and, further, through aspartate catabolism. Asparaginases are hydrolytic enzymes that produce aspartate and 
ammonium. B. subtilis expresses two periplasmic asparaginases, one, termed AnsZ, that is subjected to nitrogen control and another, termed AnsA, that is induced by asparagine or aspartate. The ans $A$ gene forms an operon with ans $B$ that encodes an aspartase. Hence, in the presence of an inducer, B. subtilis can obtain two ammonium molecules from one asparagine molecule with the concourse of a periplasmic hydrolytic enzyme, a permease, and a cytoplasmic deaminating enzyme (Fig. 2). This pathway also involves the ammonium translocator AmtB (formerly known as $\mathrm{NrgA}$ in $B$. subtilis) to incorporate into the cytoplasm the ammonium produced in the periplasm.

The enterobacteria express two asparaginases: a cytoplasmic low-affinity enzyme (the ans $A$ gene product) and a periplasmic high-affinity enzyme (the ans $B$ gene product), which are not homologous. This permits metabolism of both asparagine incorporated into the cell and extracellular asparagine. Asparagine transporters are known in bacteria, including e.g. E. coli AnsP, a member of the Amino Acid-Polyamine-Organocation (APC) Superfamily of secondary transporters, and Rhodobacter capsulatus BztABCD, an $A B C$ transporter for Glu/Gln/Asp/Asn. Aspartate produced by cytoplasmic asparaginase can be metabolized as described above for that taken up from the extracellular medium.

Regulation of expression of the genes encoding the elements for the different routes for asparagine and aspartate degradation can determine preferential utilization of these amino acids under certain conditions in particular bacteria. In E. coli, fumarate is a respiratory substrate, and under anaerobic conditions aspartate-fumarate antiporters (DcuA, DcuB) are induced that translocate fumarate to the periplasm, where it is used in respiration. Interestingly, under these conditions the periplasmic asparaginase is also induced, and asparagine is preferentially hydrolyzed in the periplasmic space, allowing the operation of the aspartatefumarate antiporter. In contrast, in Klebsiella the periplasmic asparaginase is subjected to $\mathrm{N}$ control, which is consistent with a specific role in nitrogen nutrition. The utilization of asparagine and aspartate thus illustrates the plasticity of bacteria, which can use different types of 
transporters, including secondary transporters and high-affinity $A B C$ transporters, and different enzyme localization strategies (periplasmic, cytoplasmic) to integrate assimilatory pathways and other important physiological processes of the bacterium such as respiration.

\section{Arginine}

Arginine is the amino acid richest in nitrogen, since it contains four $\mathrm{N}$ atoms within a six $\mathrm{C}$ atom skeleton. Hence, it is not surprising that arginine can be used as a source of nitrogen by many bacteria, which is reflected in the high number of different arginine catabolism pathways that can be found in the bacterial world. Whereas the nitrogen substrates discussed in this chapter generally traverse the outer membrane of Gram-negative bacteria through non-specific or lowspecificity porins, a porin specific for basic amino acids, OprD, is involved in arginine utilization in Pseudomonas aeruginosa. Arginine can be taken up into bacterial cells by arginine-specific or basic amino acid ABC transporters, of which the HisJ/ArgT-HisMQP system of Salmonella typhimurium -which is involved in the transport of His, Lys, Arg and ornithine- is a well-known example, or by arginine/ornithine antiporters of the APC superfamily. At least seven arginine catabolism pathways are found in bacteria, but only three of them utilize efficiently the four $\mathrm{N}$ atoms contained in arginine.

1. In the arginase pathway, well characterized in Bacillus, in which arginine is the second preferred nitrogen source (only after glutamine), urea is first removed from arginine by arginase (arginine ureohydrolase) with the concomitant production of ornithine (Fig. 2). Urea is then degraded by urease producing two ammonium molecules and $\mathrm{CO}_{2}$, and ornithine is further catabolized to glutamate via glutamate semialdehyde/ $\Delta^{1}-$ pyrrolyne-5-carboxylate, which involves a transamination to 2-oxoglutarate producing a second glutamate molecule. Hence, in this pathway, the four $\mathrm{N}$ atoms of arginine are conserved in two ammonium molecules and two glutamate molecules. 
2. In an alternative arginase pathway that has been well characterized in Agrobacterium, ornithine cyclodeaminase releases ammonium producing proline that is further catabolized to glutamate by the bifunctional enzyme proline oxidase, PutA. In this pathway, three ammonium molecules and one glutamate molecule are produced per arginine consumed.

3. In a variation of arginine catabolism found in some bacteria including the enterobacteria and Pseudomonads, arginine is activated by addition of succinyl-coenzyme A to produce $\mathrm{N}^{2}$ succinylarginine that follows a pathway similar to that of arginase to finally release succinate and glutamate. However, the second enzyme of this pathway, $\mathrm{N}^{2}$-succinylarginine dihydrolase, releases two ammonium molecules and $\mathrm{CO}_{2}$ instead of urea as arginase does. Hence, the arginine succinyltransferase pathway directly produces two ammonium molecules and two glutamate molecules per arginine consumed.

Other pathways make use of the arginine molecule with different purposes. Only the two most relevant of these pathways will be described here.

4. In the arginine deiminase pathway, present in many phylogenetically different bacteria, arginine is first deaminated producing ammonium and citrulline, which is then used by catabolic ornithine carbamoyltransferase to produce ornithine and carbamoyl phosphate. Whereas carbamoyl phosphate is used by carbamate kinase to produce ATP, ornithine is excreted in exchange for arginine by an arginine/ornithine antiporter. Hence, this pathway only produces one ammonium molecule per arginine utilized, but additionally produces ATP, which makes it useful as an energy-producing pathway under anaerobic conditions.

5. Arginine decarboxylase produces agmatine that is further catabolized by agmatine ureohydrolase (agmatinase, which is homologous to arginase) releasing putrescine and urea, which can be hydrolyzed by urease producing two ammonium molecules and $\mathrm{CO}_{2}$. Putrescine is a polyamine and is itself a source for synthesis of other polyamines, including spermidine. Hence, although the arginine decarboxylase pathway releases $\mathrm{N}$ in the form of urea (and further 
on ammonium), its main role appears to be the production of polyamines that are essential in many organisms.

The arginine utilization pathways outlined here illustrate the efficient use of arginine as a nitrogen source, but also its use for other metabolic purposes such as energy-conservation and polyamine biosynthesis. Utilization of arginine as a nitrogen source is tightly regulated in some bacteria such as the enterobacteria, in which expression of the ast operon encoding enzymes of the arginine succinyltransferase pathway requires nitrogen limitation and is mediated by the Ntr regulatory system. Interestingly, however, the widely-distributed repressor of arginine biosynthesis, ArgR, a transcriptional regulator of the winged helix-turn-helix (wHTH) family of DNA binding proteins that binds DNA in the presence of arginine, is also involved in expression of the ast operon in the presence of arginine. ArgR-arginine binds to the ast promoter likely facilitating the action of NtrC. The other physiological functions of arginine catabolism involve specific regulations too. Thus, for instance, expression under anoxic conditions of the arc operon encoding the proteins of the arginine deiminase pathway in Pseudomonas aeruginosa is mediated by the global regulator of anaerobic metabolism, ANR (a transcriptional regulator of the CRP/FNR family).

\section{Histidine}

Histidine is rich in nitrogen (three $\mathrm{N}$ atoms in a six $\mathrm{C}$ atom skeleton) and can be assimilated by many, although not all bacteria. The histidine assimilation pathway is relatively complex but well conserved among bacteria. It involves histidine uptake and further degradation in four or five successive enzymatic steps, producing one glutamate and one or two ammonium molecules, respectively, per histidine molecule consumed. Because the imidazole side chain of histidine has a $\mathrm{pK}_{\mathrm{a}}$ of 6.0 , at a neutral $\mathrm{pH}$ histidine can be taken up through both neutral and basic amino acid $A B C$ uptake transporters. Thus, the $A B C$ basic amino acid transporter HisJ/ArgT-HisMQP of Salmonella typhimurium is involved in histidine uptake. Additionally, there are histidine-specific 
transporters such as the HutT permease of the Pseudomonads, a secondary transporter of the APC family.

Histidine is deaminated by histidase (the hutH gene product) producing ammonia and urocanate, which is subjected to two successive hydration steps mediated by urocanase (the hutU gene product) and imidazolone propionate hydrolase (the hutl gene product) forming imidazole propionate and formimino glutamate, respectively. In some bacteria (e.g., those of the genera Bacillus and Klebsiella), formimino glutamate is hydrolyzed by formimino glutamate hydrolase (the hutG gene product) releasing glutamate and formamide, which is not further catabolized, being instead excreted. In these bacteria, therefore, the pathway renders one glutamate molecule and one ammonium molecule per histidine consumed (Fig. 2). In other bacteria (e.g., those of the genera Pseudomonas and Streptomyces), formimino glutamate deiminase (the hutF gene product) produces ammonium and formyl glutamate, which is hydrolized by formylglutamate hydrolase (the product of a hutG gene that is not homologous to that of Bacillus or Klebsiella) releasing formate and glutamate. Hence, in these bacteria two molecules of ammonium and one molecule of glutamate are obtained per histidine consumed (Fig. 2), which represents an optimal efficiency in the utilization of histidine as a nitrogen source.

The hut genes encoding histidine catabolism enzymes and a transporter-encoding gene, hutT, are frequently clustered together constituting one or several transcriptional units. A gene, hutC, encoding a transcriptional repressor is also frequently found in the hut gene cluster. Because the biosynthesis of histidine is energetically expensive, the expression of the catabolic hut genes is subjected to tight regulation to avoid a futile cycle. Thus, the hut genes are only expressed if excess histidine is available and a shortage of nitrogen or carbon is perceived in the cells. Urocanate (the first catabolic product) is an inducer blocking the HutC repressor activity, thus ensuring that the hut genes are expressed only if histidine is abundant. An important variation in the mechanism of induction is found in Bacillus, where histidine-promoted antitermination mediated by a protein termed HutP is in operation. Additionally, hut genes are 
subjected to catabolite repression and to $\mathrm{N}$ control. In enterobacteria, catabolite repression implies CAMP-CRP-mediated activation of expression of the hut genes, and their expression under nitrogen deficiency requires the Nac transcription factor. C- and N-regulation of hut genes in other bacteria such as those of the genera Pseudomonas or Streptomyces is carried out by their corresponding global regulatory systems.

\section{Atrazine and cyanuric acid}

Atrazine is very rich in nitrogen (five $\mathrm{N}$ atoms in an eight $\mathrm{C}$ atom skeleton), and an atrazinemineralization pathway is encoded in plasmids found in some Pseudomonads, which make such organisms capable of using this xenobiotic as a nitrogen source. Cyanuric acid, an intermediate in the atrazine degradation pathway, can also be used as nitrogen source in these bacteria. Atrazine mineralization frequently occurs via a hydrolytic pathway that proceeds through the sequential elimination of the chlorine, ethylamino and isopropylamino substituents, to yield cyanuric acid that is cleaved and mineralized to $\mathrm{CO}_{2}$ and ammonia (Fig. 2). Thus, mineralization of atrazine renders one ethylammonium and one isopropylammonium molecule, on one hand, and three ammonium molecules that can be directly incorporated into carbon skeletons, on the other. Not surprisingly, the capability to use atrazine is only expressed under nitrogen deficiency, but this is based in regulation of only part of the atrazine degradation pathway. Whereas the genes encoding the enzymes that catalyze the first three steps in the pathway $(a t z A, a t z B, a t z C)$ are scattered in the atrazine-catabolism plasmid and appear to be constitutive, the genes encoding the enzymes that catalyze the last three steps of the pathway (those involved in degradation of cyanuric acid) constitute an operon (atzDEF) whose expression is tightly regulated. The atzDEF promoter is a standard $\sigma^{70}$-dependent promoter that is activated by AtzR, a LysR-type transcriptional regulator that is encoded just upstream, divergently from the operon. The $a t z R$ gene is expressed from a $\sigma^{54}$-dependent promoter whose activation requires the general Ntr system of the bacterium, thus linking the capability to mineralize 
atrazine to nitrogen deficiency. The AtzR protein additionally requires cyanuric acid as a coactivator, and its activity is further enhanced by uridylylated GInK. In addition to the only partial regulation of the atrazine degradation pathway, it is of interest that whereas the plasmid bearing the genes for atrazine/cyanuric acid degradation also has genes encoding an $A B C$ transporter for cyanuric acid, the presence of a transporter for atrazine is unclear. Hence, atrazine is a nitrogen source whose utilization has likely involved the integration of genes specific for its degradation down to cyanuric acid with a pre-existing pathway for the utilization of this compound. Thus, degradation of atrazine and cyanuric acid exemplifies the adaptability of bacteria that have evolved to use xenobiotics as nitrogen nutrients.

\section{The assimilation of nitrate}

Nitrates are present at low concentrations (micromolar or below) in natural habitats and in the oceans. However, nitrates are relatively abundant in local areas, especially those that hold extensive agricultural activity, because the use of excessive fertilization often leads to nitrate leakage into the environment. Nitrate is an excellent nitrogen source for many bacteria and fungi, algae and plants, and its assimilation involves entrance into the cell by an active transporter and its intracellular reduction to ammonium by two sequential reductive reactions, with ammonium being finally incorporated into organic material (Fig. 2).

\section{Nitrate uptake}

Two types of active transport systems for nitrate, which can also accept nitrite, have been described in bacteria: ABC-type transporters and MFS permeases. Whereas the nitrate/nitrite transporters described in several bacteria (such as $K$. oxytoca) conform to the basic structure of $A B C$ transporters, in cyanobacteria the Nrt transport system presents some peculiarities. First, the binding protein, NrtA, is anchored to the periplasmic side of the cytoplasmic membrane by 
post-transcriptional processing (including lipidation) by signal peptidase II and, second, the ATPase domain is made of two different subunits: NrtD, a standard ATPase subunit, and NrtC, a long protein with an $\mathrm{N}$-terminal part similar to NrtD and a C-terminal part homologous to NrtA. The permease domain of Nrt is made of two NrtB subunits, each providing 5-transmembrane helices.

Major facilitator superfamily (MFS) permeases are made of dimers of a single gene product that normally contains 12 transmembrane segments and couple substrate transport or exchange through the cytoplasmic membrane to the dissipation of an electrochemical gradient. The Bacillus subtilis or Paracoccus denitrificans NasA, the Synechococcus sp. PCC 7002 NrtP and the Trichodesmium sp. WH9601 NapA belong to this family of transporters and couple nitrate/nitrite transport to a $\mathrm{H}^{+}$or $\mathrm{Na}^{+}$gradient.

\section{Nitrate reduction}

Intracellular nitrate is first reduced to nitrite in a $2 e^{-}$-transfer reaction catalyzed by nitrate reductase, and the resulting nitrite is then reduced to ammonium in a $6 \mathrm{e}^{-}$-transfer reaction catalyzed by nitrite reductase. In spite of this general scheme, the reductases largely diverge in structure and electron transfer paths depending on the organism. In cyanobacteria, which are characterized by a photoautotrophic mode of life relying on oxygenic photosynthesis, both nitrate reductase and nitrite reductase utilize photosynthetically reduced ferredoxin or flavodoxin as the electron donor. In most heterotrophic bacteria, and some anoxygenic phototrophs such as Rhodobacter capsulatus, both reductases accept electrons from reduced pyridine nucleotides (NADH), although some exceptions are found. For example, the nitrate reductase from Azotobacter vinelandii uses reduced flavodoxin, and the nitrate reductase from the chemolithoautotroph Hydrogenobacter thermophilus and the nitrate and nitrite reductases from the archaeon Haloferax mediterranei utilize reduced ferredoxin as electron donor. 


\section{Nitrate reductase}

The cyanobacterial nitrate reductase, as of that of Synechococcus elongatus, is a monomeric enzyme of ca. $80 \mathrm{kDa}$, the product of the narB gene, containing one [4Fe-4S] cluster, which receives electrons from the external electron donor, and a molybdenum-bis-molybdopterin guanine dinucleotide (Mo-bisMGD) cofactor, which represents the active site for nitrate reduction (Fig. 6). In contrast, NADH-dependent nitrate reductases frequently contain at least an additional reductase subunit, with FAD and sometimes Fe-S clusters, besides the MobisMGD-containing catalytic subunit, which also includes one [4Fe-4S], and sometimes a [2Fe2S], cluster. In the case of Klebsiella oxytoca, nitrate reductase is constituted by a 92-kDa catalytic subunit (NasA -please note, different from B. subtillis NasA above), including a MobisMGD, one [4Fe-4S] and one [2Fe-2S] cluster, and a reductase subunit of $43 \mathrm{kDa}$ (NasC), which contains FAD and transfers electrons from NADH to the catalytic subunit. Cyanobacterial Fddependent, $H$. thermophylus Fd-dependent and the catalytic subunit of NADH-dependent nitrate reductases, are all homologous proteins. $\quad$ <Fig. 6 near here>

\section{Nitrite reductase}

Although appreciable differences can be found between ferredoxin- and NADH-dependent nitrite reductases, all of them exhibit considerable sequence and structural similarities as well. In particular, all nitrite reductases studied to date include [4Fe-4S] and siroheme groups similar to that of the sulfite reductase hemoprotein from enteric bacteria. The cyanobacterial enzymes are monomers, generally of ca. $55 \mathrm{kDa}$ (NirA), where electrons from ferredoxin are transferred to the $[4 \mathrm{Fe}-4 \mathrm{~S}]$ cluster and then the siroheme (Fig. 6). Cyanobacterial nitrite reductase has been conserved in the chloroplasts and, hence, is an enzyme of universal relevance in photosynthetic organisms. Bacterial NADH-nitrite reductases may be monomeric, such as that of $K$. oxytoca (NasB), or, as in the case of $B$. subtilis, dimeric and constituted by a large catalytic subunit (NasD) and a small subunit (NasE) with similarity to the carboxy-terminal part of monomeric NasB. Both 
NasB and NasD include non-covalently bound FAD and two [2Fe-2S] clusters besides [4Fe-4S] and siroheme groups, the latter as found in cyanobacterial nitrite reductase.

\section{Genetics and regulation}

In cyanobacteria, the genes for the structural components of the nitrate and nitrite assimilation system (nitrate reductase, nitrite reductase and nitrate/nitrite transporter) are frequently clustered together with the order nirA-transporter gene(s)-nirB, although some variants of this arrangement can also be found. In the well-studied cyanobacteria Synechococcus elongatus and Anabaena sp. PCC 7120, these genes constitute an operon that is preceded by additional genes involved in modulation of either the expression of the nirA operon or the activity of its encoded elements. Outside of the cyanobacteria, a cluster of nitrate/nitrite assimilation genes has been identified in all the nitrate-assimilating bacteria studied to date, which sometimes also includes regulatory genes and genes for electron donors and synthesis of the nitrite reductase cofactor siroheme or the nitrate reductase cofactor Mo-bisMGD.

Nitrate (and nitrite) assimilation is a costly process due to the energetic requirement for active transport, the high requirement for reducing equivalents and the synthesis of complex reductase cofactors. Thus, in most bacteria, nitrate assimilation is subjected to global $\mathrm{N}$-control circuits (see $\mathrm{N}$-control section). In addition, frequently, pathway-specific regulators -often encoded in the nitrate/nitrite assimilation gene cluster- influence the expression of nitrate assimilation genes, in some cases eliciting positive responses to the availability of nitrate or nitrite.

In cyanobacteria, nitrate and nitrite assimilation genes are under global $\mathrm{N}$ control exerted by the transcriptional regulator NtcA, and in the case of Synechococcus, the activity of the Nrt active transporter for nitrate and nitrite has been shown to be regulated by $P_{\|}(G \ln B)$, which in cyanobacteria is phosphorylated in response to low 2-oxoglutarate levels. In addition, expression of the nirA operon is subjected to pathway-specific regulation by the LysR-type 
transcription factor $\mathrm{NtcB}$. NtcB exerts a positive effect on the expression of nitrate assimilation genes in the absence of ammonium, which is of high magnitude in the case of Anabaena sp. PCC 7120. In this case, NtcB has been shown to bind DNA in the promoter region of the nirA operon, close to the binding site for NtcA, and the expression of the $n t c B$ gene is itself activated by NtcA.

In many bacteria, responses to the presence of nitrate or nitrite appear to be mediated by transcription anti-termination mechanisms. In $K$. oxytoca and $A$. vinelandii, expression of the nas operon is regulated by the general $\mathrm{N}$-control Ntr system. In addition, the factors NasR or NasT, respectively, bind the leader sequence of the nas operon mRNA avoiding premature termination, thus promoting transcription completion in the presence of nitrate or nitrite. NasT acts together with NasS, a protein homologous to cyanobacterial NrtA, which may represent a cytoplasmic nitrate/nitrite sensor.

\section{Nitrogen Fixation}

In order to enter the biosphere, atmospheric $\mathrm{N}_{2}$ must be first reduced to a form that can be readily assimilated by organisms, usually ammonium. This reduction is known as nitrogen fixation. Nitrogen fixation is a highly energy-demanding process, given that the triple bond in dinitrogen is very stable and difficult to break down. As a direct result of the inertness of dinitrogen, nitrogen can become a limiting element in ecosystems and, particularly, in agriculture, where fixed nitrogen is the main component of fertilizers.

Biological nitrogen fixation is carried out exclusively by prokaryotes, both bacteria and archaea, collectively known as diazotrophs. Diazotrophy is not limited to any specific group, and it appears to be widely, but haphazardly, distributed throughout different prokaryotic groups. This distribution, together with the high conservation of nitrogen fixation systems (see later in this section) and their extreme oxygen sensitivity, suggest that diazotrophy is a very ancient capability. Ecologically, diazotrophy is also widely distributed. Substantial rates of nitrogen fixation have been observed in terrestrial and aquatic (both freshwater and oceanic) 
ecosystems, where diazotrophic prokaryotes are often in close, symbiotic associations with eukaryotic organisms, from unicellular algae to fungi, plants and animals.

\section{Nitrogenases}

Diazotrophic organisms carry out biological nitrogen fixation under normal conditions of pressure and temperature, compatible with life, through the catalytic action of a complex enzyme, nitrogenase. Although some nitrogenase variants exist, especially regarding the complement of transition metals at their active center, the available information on their structure and organization, as well as on their assembly and biochemistry, denotes a striking conservation of nitrogenases among diazotrophs.

Nitrogenases catalyze the reduction of dinitrogen to ammonia at the expense of ATP, protons and reducing power, according to the equation:

$\mathrm{N}_{2}+8 \mathrm{H}^{+}+8 \mathrm{e}^{-}+16 \mathrm{MgATP} \rightarrow 2 \mathrm{NH}_{3}+\mathrm{H}_{2}+16 \mathrm{MgADP}+16 \mathrm{P}_{\mathrm{i}}$

where ammonia is in the protonated ammonium form in solution.

Two striking characteristics of this equation are its high energetic cost, in terms of ATP and reducing power, and the stoichiometric synthesis of one molecule of hydrogen gas per molecule of dinitrogen reduced. The latter is a source of energy inefficiency in the reaction, since $2 \mathrm{e}^{-}$and $4 \mathrm{MgATP}$ are used to make $\mathrm{H}_{2}$ that can be lost as gas. This is an unavoidable outcome of the complex enzymatic mechanism required to reduce dinitrogen, whereby electrons must be pumped in rapid succession onto a dinitrogen molecule immobilized at the active center that undergoes stepwise reductions through short-lived, unstable intermediates. Nonetheless, many diazotrophs express hydrogenases that can oxidize the hydrogen produced in the nitrogenase reaction, recovering electrons and producing ATP.

The structural and biochemical characteristics have been studied especially in nitrogenases that contain the transition metal molybdenum (Mo-nitrogenases). Other, less common and less studied, variants, replace vanadium (V-nitrogenases) for molybdenum. A third 
type of nitrogenase contains iron instead of Mo or V. Since all of the above variants also contain additional Fe, the latter are known as Fe-only nitrogenases.

Mo-nitrogenase is actually a complex of two metalloenzymes. The largest, dinitrogenase (also known as MoFe protein) is a ca. $250 \mathrm{kDa}$ heterotetramer $\left(\alpha_{2} \beta_{2}\right)$ organized as two $\alpha \beta$ heterodimers (Fig. 7A). Each dimer contains a unique iron-molybdenum cofactor (FeMo-co) on the $\alpha$ subunit, the active site of substrate binding and reduction, and a unique Fe-S cluster, the P cluster at the $\alpha-\beta$ subunit interface. The smaller enzyme, dinitrogenase reductase (also known as Fe protein) is a ca. $46 \mathrm{kDa} \alpha_{2}$ homodimer (Fig. 7A). Each subunit contains an ATP/ADP binding site and both subunits are bridged by a $4 \mathrm{Fe}-4 \mathrm{~S}$ cluster.

The role of dinitrogenase reductase is to deliver electrons to dinitrogenase, one at a time. In turn, dinitrogenase reductase is reduced by a cellular source of high-energy electrons, often ferredoxins or flavodoxins, that varies among diazotrophs and is often a reflection of its particular lifestyle. Once reduced, dinitrogenase reductase transiently associates with dinitrogenase and hydrolizes 2 molecules of ATP to ADP in order to transfer one electron to dinitrogenase. After transfer, the oxidized enzyme dissociates and replaces the ADPs by ATPs, getting ready to be reduced again. Electrons received by dinitrogenase are stored in the protein and eventually transferred to the substrate in FeMo-co through the P clusters. Therefore, eight interlocking catalytic cycles of dinitrogenase reductase are required per cycle of dinitrogenase in what is one of the slowest enzymatic rates (ca. 1 cycle per second). Given these characteristics, and the fact that, despite the availability of high resolution structures, bound substrate and intermediates have not been unequivocally visualized, it is not surprising that details of the nitrogenase mechanism have yet to be ascertained.

The two complex metal cofactors of dinitrogenase, FeMo-co and P clusters, are unlike any other found in biological systems. Despite the fact that FeMo-co (and probably P clusters) can be extracted from the enzyme in active form, they are extremely labile, and their structure has only been solved within the enzyme. FeMo-co contains a [4Fe-3S] subcluster and a [3Fe-Mo- 
3S] subcluster separated by a C atom, probably a carbide, and a molecule of (R)-homocitrate coordinated to Mo through its 2-hydroxy and 2-carboxyl groups. FeMo-co synthesis and assembly require a large number of specific proteins, and its mechanism has been unraveled through the use of sophisticated in vitro systems with purified components and precursors. $\mathrm{P}$ clusters are [8Fe-7S] clusters that originate by specific, in situ reductive fusion of two standard [4Fe-4S] clusters. Both FeMo-co and P clusters confer oxygen sensitivity to dinitrogenase, which is rapidly inactivated and denatured upon exposure to air. Even higher oxygen sensitivity has been observed with dinitrogenase reductase, despite the fact that its [4Fe-4S] cluster is similar to clusters present in other, oxygen-resistant proteins.

Although fragmentary, the available evidence suggests that V-nitrogenases and Fe-only nitrogenases are evolutionarily related to the more widespread Mo-nitrogenases, that their general architecture, synthesis and mechanism are similar, and that they may reflect adaptations to diazotrophy under conditions where Mo may become limiting for nitrogen fixation. In this regard, several considerations could be taken into account: a) the catalytic inefficiency of nitrogenase (see above) is often compensated by the synthesis of very high levels of the enzyme, that can amount to over $20 \%$ of the total cell protein; b) Mo-depleted ecosystems exist, such as some acidic soils and open oceanic waters; c) the catalytic efficiency of V-and Fe-nitrogenases is lower than that of Mo-nitrogenases; and d) one or both of these "alternative" systems can be found in diazotrophs that also possess a Mo-nitrogenase. In those cases, the more efficient Mo nitrogenase is expressed when Mo is available, and the several systems can share some of their accessory components, such as those related to metal cofactor synthesis.

\section{Genetics and regulation}

The genetic derminants for nitrogenase and nitrogenase synthesis have been studied in a few model systems, notably K. pneumoniae and A. vinelandii (Fig. 7B). Comparative DNA sequence 
analyses, stimulated by the recent explosion in prokaryotic genome sequencing, have shown that many of the nif (nitrogen fixation) determinants, notably those encoding structural proteins, are strongly conserved in diazotrophs. Analysis of their functions has been facilitated by their organization in tight gene clusters containing co-regulated operons. The number of nif genes in diazotrophs is variable, from over 30 in A. vinelandii, 20 in K. pneumoniae, and down to nine in Firmicutes (Fig. 7B) and some archaea, with a minimum set of six nif genes conserved throughout. These include nifH, nifD, nifK, nifE, nifN, and nifB. The first three encode the dinitrogenase reductase structural subunit and the $\alpha$ and $\beta$ subunits of dinitrogenase, respectively, while the last three are involved in FeMo-co synthesis: NifE and NifN are structural homologues of NifD and NifK, respectively, and form a $\alpha_{2} \beta_{2}$ heterotetramer that acts as a scaffold on which FeMo-co is assembled from a precursor cluster (NifB-co) synthesized by NifB. Other nif genes are involved in Mo metabolism, synthesis of Fe-S cluster precursors and homocitrate, on one hand, and nitrogenase assembly and maturation, electron transfer to nitrogenase and regulation, on the other. Some of these functions, such as the first three, can be provided by the housekeeping cellular machinery, although it is not uncommon to find nifspecific versions of those, such as in the case of Fe-S cluster synthesis, in view of the demands that high levels of nitrogenase synthesis can impose on housekeeping elements.

As is the case with electron transfer to nitrogenase -highly dependent upon specific metabolic lifestyles- different models of nitrogen fixation regulation exist, so as to adapt nitrogen fixation expression to specific cellular contexts. Besides the high energy requirements, the enzyme is strongly and irreversibly inactivated by oxygen. Therefore, it is not surprising that diazotrophs prefer to use fixed nitrogen sources over dinitrogen, and that nitrogenase is usually tightly regulated by the nitrogen and oxygen status of the cell, either directly or indirectly. Regulation of nitrogen fixation occurs mainly at the transcriptional level rather than at the activity level. Contrary to many biosynthetic enzyme systems, nitrogenase is not subject to endproduct inhibition, and oxygen simply inactivates the enzyme irreversibly. 
Transcriptional regulation of nitrogen fixation has been studied in detail in proteobacteria (K. pneumoniae, A. vinelandii or the Rhizobia). Promoters of nif genes are dependent on an alternative sigma factor $\left(\sigma^{54}\right)$ that recognizes consensus sequences at positions -24 and $-12 . \sigma^{54}$-RNA polymerase holoenzyme can initiate transcription only after $\sigma^{54}$ interacts with a transcriptional activator bound to upstream enhancer sequences. In the case of nif promoters, this activator is NifA, a homodimeric protein that belongs to the ATPase AAA+ superfamily and that, using ATP hydrolysis, determines a conformational change in $\sigma^{54}$ that results in the formation of a transcriptionally competent RNA polymerase-promoter open complex. This strategy is advantageous in that it ensures that no low-level transcription can occur in the absence of NifA, and in that it provides a target, NifA, for signal transduction. In some organisms, notably the Rhizobia, NifA is able to sense the oxygen status directly, becoming inactivated under aerobic conditions, and even the $\mathrm{N}$ status. In others, such as in $K$. pneumoniae and $A$. vinelandii, nifA is cotranscribed with nifL. The NifL protein has the ability to bind to NifA, inactivating it in response to environmental signals, such as the redox, $\mathrm{N}$ and $\mathrm{C}$ status of the cell. In that respect, the NifLA pair resemble two-component regulatory systems. However, although it is possible that they may have evolved from such a system (the identifiable remnants of a histidine-protein kinase domain are present in NifL), their ability to integrate these disparate environmental signals, not only within NifL, but in some cases also within NifA, makes this an unusual regulatory pair. Transcription of nifA (or nifLA) can, in turn, be subjected to environmental signal control in a cascade-like manner, thus ensuring an amplification of the signal compatible with the need for a tight, highly responsive regulation of nitrogen fixation.

This specific picture of nitrogen fixation regulation, drawn from studies of proteobacteria, is not shared by many other diazotrophs. Thus, although detailed knowledge of specific regulatory mechanisms is lacking in many cases, proteins homologous to NifA proteins are missing from the genomes of diazotrophs as diverse as the archaea and the Firmicutes, among others. However, the basic tenets of expression in the absence of fixed nitrogen sources 
and oxygen still hold in most cases. The nif genes from methanogenic archaea and Firmicutes appear to be regulated by transcriptional repression, rather than induction. Cyanobacteria also lack NifA and appear to use a redox-sensitive transcriptional activator, CnfR. In strains without heterocysts (cells specialized in $\mathrm{N}_{2}$ fixation), $\mathrm{CnfR}$ is expressed under $\mathrm{N}$ starvation, while in heterocyst-forming strains, it is expressed only in heterocysts (see section "The heterocyst").

A further aspect of regulation concerns the alternative $\mathrm{V}$ - and Fe-nitrogenases, especially when they coexist with a Mo-nitrogenase system, such as in $A$. vinelandii and the cyanobacterium Anabaena variabilis. A. vinelandii is unique in containing all three, Mo-, $\mathrm{V}-$, and Fe-only nitrogenase systems. The alternative systems are regulated by NifA orthologues, $\mathrm{VnfA}$ and AnfA, respectively, in response to $\mathrm{N}$ and $\mathrm{O}_{2}$ signals, and by a further, hierarchical regulation with preference for Mo-nitrogenase $>$ V-nitrogenase $>$ Fe-only nitrogenase. Rather than sensing the transition metal directly, regulation appears to respond to the efficiency of the nitrogenase system in a not well-understood cross-talk that implicates NifA, VnfA and AnfA. A. variabilis contains two Mo-nitrogenases and one V-nitrogenase. The main Mo- and the V-nitrogenase systems are hierarchically expressed in heterocysts, while the second Mo-nitrogenase is only expressed under anaerobic conditions, and then in all the cells in the filament.

Finally, it is worth noting that a post-transcriptional regulatory mechanism of nitrogenase activity operates in some $\alpha$-proteobacteria, such as Azospirillum brasilense, and purple photosynthetic bacteria. In these bacteria, dinitrogenase reductase is reversibly inactivated by ADP-ribosylation in response to ammonium or lack of energy (darkness in photosynthetic bacteria or anaerobiosis in A. brasilense).

\section{Mechanisms of protection of nitrogenase against oxygen}

The extreme oxygen sensitivity of nitrogenase proteins and cofactors is probably a reflection of the fact that they evolved before the Great Oxygenation Event. Nonetheless, the physiological and ecological advantages afforded by the ability to fix nitrogen in fixed $\mathrm{N}$-depleted 
environments, even in the presence of air, have resulted in the acquisition of a wide range of adaptations to protect nitrogenase from oxygen. The simplest case is that of the obligate anaerobes, such as archaea and the clostridia. These diazotrophs cannot live in the presence of oxygen even if they are not fixing nitrogen, therefore no special adaptations of nitrogenase to oxygen is required or has been selected. Facultative anaerobes, such as enterobacterial diazotrophs, can fix nitrogen only under fermentative conditions, but their respiratory systems have been shown to help scavenge oxygen by using it as electron acceptor.

A large number of chemoorganotrophic, diazotrophic bacteria are dependent on respiration with oxygen as terminal electron acceptor to obtain energy, and can only fix nitrogen under decreased oxygen tension, where the combined effect of physical barriers to oxygen diffusion and respiratory consumption of the diffused oxygen result in near-anoxic conditions compatible with nitrogenase expression and activity. This is the case of the Rhizobia, that associate with legumes to fix nitrogen within a special root organ, the nodule, where root and bacterial respiration contribute to decrease oxygen levels. However, the obligatory need for oxygen as terminal electron acceptor in the Rhizobia has resulted in specific molecular adaptations of the symbiosis, such as the accumulation of a legume hemoglobin, leghemoglobin, that provides the bacteria with a steady supply of oxygen at low levels, or the expression of a high-oxygen-affinity terminal respiratory oxidase that allows the bacteria to obtain energy at these low oxygen levels.

A. vinelandii exemplifies the best-studied case of strictly aerobic diazotrophy in unicellular bacteria. Complete protection of nitrogenase against oxygen is provided, again, by a combination of structural oxygen diffusion barriers (cell envelopes, exopolysaccharides) and respiratory protection (increased respiration mediated by several redundant terminal oxidases with high capacity and high affinity towards oxygen), which together result in an adequate decrease in dissolved oxygen levels and diffusion rates. It is worth noting that one of these high capacity oxidases -a quinol oxidase- can become uncoupled from ATP synthesis and thus work 
as a combustion machine, provided that reducing power is not limiting. A further adaptation to diazotrophy under air is the presence in $A$. vinelandii of the Fe/S II protein, which can bind to nitrogenase forming an oxygen-resistant complex that can preserve the integrity of nitrogenase under transitory episodes of increased oxygen.

\section{Protection in oxygenic phototrophs (Cyanobacteria)}

In cyanobacteria, the issue of protection of the nitrogenase system from oxygen is specially demanding because, as oxygenic phototrophs, they not only have to cope with ambient oxygen but also with that produced intracellularly by oxygenic photosynthesis. Nevertheless, the capacity to fix $\mathrm{N}_{2}$ is widespread in the cyanobacterial phylum, and different strains have evolved different strategies to fix $\mathrm{N}_{2}$ in oxic environments, although some strains can do so only under micro-oxic or anoxic conditions. As an example of the latter, cyanobacteria of the genus Oscillatoria can fix $\mathrm{N}_{2}$ while performing $\mathrm{H}_{2} \mathrm{~S}$ - and photosystem I-dependent anoxygenic photosynthesis under anaerobic conditions. Besides that, well known strategies for protection against intracellular $\mathrm{O}_{2}$ are based on temporal or spatial separation of the processes of $\mathrm{N}_{2}$ fixation and oxygenic photosynthesis. Thus, in many filamentous and unicellular cyanobacteria, $\mathrm{N}_{2}$ fixation is restricted to the dark periods when subjected to a diel cycle, and likely under natural conditions (as shown for the marine unicellular organism Chroccosphaera sp.). In the dark period, $\mathrm{N}_{2}$ fixation can coincide with active respiration, which has a dual function in provision of energy and oxygen scavenging. As an example, in the unicellular Cyanothece sp., photosynthesis, ATP synthase, $\mathrm{CO}_{2}$-fixation and glycogen biosynthesis genes are expressed in the light periods, whereas glycogen degradation, respiration and nif genes are expressed in the dark periods. This pattern of expression involves circadian regulation. In contrast, in the filamentous cyanobacterium Trichodesmium sp., a globally-relevant $\mathrm{N}_{2}$ fixer in the oceans, $\mathrm{N}_{2}$ fixation peaks in the light periods during diel cycles. Although the mechanism of nitrogenase protection is not clear, it is debated whether $\mathrm{N}_{2}$ fixation is confined to cells in a central stretch 
of the filament that would have reduced oxygen content, perhaps aided by temporal segregation of $\mathrm{CO}_{2}$ and $\mathrm{N}_{2}$ fixation during the day. Further specialization is found in some filamentous cyanobacteria, included in a defined phylogenetic clade, that can produce heterocysts, which are cells specialized for the fixation of $\mathrm{N}_{2}$. In these strains, heterocysts are the only cells of the filament that, under oxic conditions, express nitrogenase (Fig. 8).

$<$ Fig. 8 near here $>$

\section{The heterocyst}

The differentiation of a vegetative cell into a heterocyst takes place in response to $\mathrm{N}$ deprivation and involves extensive structural and biochemical changes to provide suitable sites for efficient $\mathrm{N}_{2}$ fixation. Morphological changes that take place during differentiation include the deposition of specific glycolipid and polysaccharide cell envelope layers to limit gas, including oxygen, penetration into the cell cytoplasm. Indeed, it has been proposed that the main path for $\mathrm{N}_{2}$ entrance into the heterocyst is a differentiated, membrane protein structure at the heterocyst poles that could modulate gas diffusion rates. Also, conspicuous reorganization of the photosynthetic membranes, which is concomitant with loss of photosystem II function (and thus of oxygenic photosynthesis), takes place during differentiation.

Biochemically, the photoautotrophic metabolism of the vegetative cell is turned into a heterotrophic metabolism in the heterocyst. This transition is based on the loss of photosynthetic $\mathrm{CO}_{2}$ fixation (which is catalyzed by ribulose-bisphosphate carboxylase/oxygenase [RubisCo]), expression of a specific invertase for sucrose catabolism, increased levels of the oxidative pentose phosphate cycle enzymes, and expression of specific terminal respiratory oxidases. Indeed, the heterotrophic metabolism of heterocysts relies on sugars donated by the neighboring vegetative cells, which also provide carbon skeletons for the incorporation of the ammonium resulting from $\mathrm{N}_{2}$ reduction. In turn, heterocysts transfer fixed nitrogen in the form of amino acids (including a dipeptide, $\beta$-aspartyl-arginine) to the vegetative 
cells. Thus, under diazotrophic conditions the organismic unit of these cyanobacteria is a filament constituted by different cell types with specialized metabolic functions that exchange nutrients and regulatory molecules (see later in this section), representing true pluricellular bacteria (Fig. 8).

At the genetic level, heterocyst differentiation involves the sequential action of the products of multiple regulatory and structural genes, leading to the establishment of a gene expression pattern differing conspicuously from that taking place in the vegetative cells. Two transcription factors are required for triggering differentiation: the global N-regulator NtcA (see $\mathrm{N}$-control section), and HetR. At the initiation of differentiation, basal levels of NtcA are activated by the increased levels of 2-oxoglutarate that result from an increased C-to- $\mathrm{N}$ cellular balance, and the genes $n t c A$ and hetR are activated in a mutually- and self-dependent manner, which leads to the establishment of high levels of both regulators. Remarkably, activation of the expression of the genes encoding these two main regulators, as well as of many of their regulated genes, takes place in the specific cells of the filament engaged in differentiation. Thus, many genes involved in heterocyst differentiation and function present spatiotemporal specificity for activation, including the nif genes (Fig. 7B), which are expressed only in mature heterocysts. Molecular events contributing to this pattern include the remarkable occurrence of complex promoter regions for many of those genes, which bear several successive promoters with different requirements for NtcA and HetR, and the participation of secondary regulators, which are expressed at precise stages during differentiation, some of which increase the expression or activity of NtcA or HetR (Fig. 9). <Fig. 9 near here>

Heterocysts are not randomly-distributed in the cyanobacterial filament. In the case of strains such as those of the genera Anabaena or Nostoc, heterocysts are flanked by stretches of ca. 10 vegetative cells, an adequate pattern to fulfill the nutritional interdependence between the two cell types. The PatS morphogen is a key element in establishing the heterocyst distribution pattern. The patS gene is induced early in differentiating cells and encodes a primary 
product of 17 amino acids that is processed to render the active molecule (a C-terminal peptide of likely seven residues), which is a HetR (and thus a differentiation) inhibitor. Indeed, PatS is transferred from source cells, where the patS gene is expressed, to neighboring cells, setting a concentration gradient with higher levels in the cells adjacent to the source cell, within which the concentration remains negligible, and diminishing with distance from there on (Fig. 9). The gradient of PatS peptide away from the heterocyst appears to create a gradient of HetR to determine the pattern of cellular differentiation in each filament.

\section{Nitrogen storage}

Although scarcely studied, nitrogen storage could take place in different bacteria. Only one specific nitrogen reservoir is known, the cyanophycin granules that will be discussed here. Nonetheless, the general protein pool of a bacterium, or specific proteins such as phycobiliproteins in the case of cyanobacteria, can constitute nitrogen reservoirs made available by protein turnover under nitrogen deficiency.

In most cyanobacteria, cyanophycin represents a dynamic reservoir of nitrogen that is accumulated in the form of cytoplasmic granules. Cyanophycin accumulates under conditions of unbalanced growth, e.g., under phosphate or sulfur starvation or high $\mathrm{CO}_{2}$ supply. In diazotrophic strains that temporarily separate oxygenic photosynthesis and $\mathrm{N}_{2}$ fixation, cyanophycin accumulation may coincide with the periods of $\mathrm{N}_{2}$ fixation. In filamentous heterocyst-forming strains, cyanophycin is conspicuously accumulated at the polar regions of the heterocysts (Fig. 8), representing a pool of fixed $\mathrm{N}$ en route to the vegetative cells. First thought to belong only to cyanobacteria, genes encoding cyanophycin-metabolism enzymes have later been identified in the genomic sequence of many other bacteria with different metabolic options including phototrophy, aerobic or anaerobic respiration, chemolithoautotrophy or fermentation. As in cyanobacteria, cyanophycin is accumulated under phosphate limitation in, e.g., Acinetobacter sp. Interestingly, cyanophycin is considered a source 
of products with potential interest in the chemical and pharmacological industries (biopolymers, natural food additives, therapeutic amino acid supplements), and projects aimed at increasing the accumulation of cyanophycin in several bacteria, as well as the expression of cyanophycin synthesizing enzymes in heterologous hosts, including yeast and plant plastids, have been developed.

\section{Cyanophycin metabolism}

Cyanophycin is made of polypeptide chains of variable length that contain arginine residues linked to a polyaspartate backbone in a nearly 1:1 (Arg:Asp) ratio. The cyanophycin polypeptides are water insoluble and form granules. Cyanophycin is non-ribosomally synthesized by cyanophycin synthetase, which catalyzes both the elongation of the backbone by addition of LAsp forming an $\alpha$-peptide bond, and the addition of L-Arg to the $\beta$-carboxyl group of Asp making an isopeptide bond. Both reactions require ATP. The enzyme is a homodimer, the product of the cphA gene, with two predicted catalytic sites: one C-terminal site for the incorporation of Asp, and one N-terminal site for the addition of Arg. Cyanophycin degradation takes place in two sequential steps. First cyanophycinase, a C-terminal exopeptidase, a dimer of the product of the $c p h B$ gene, excises a $\beta$-Asp-Arg dipeptide. The dipeptide is further hydrolyzed to the constituent amino acids by isoaspartyl dipeptidase, which is a dimer of two different subunits resulting from autoproteolytic cleavage of the precursor gene product, and which is similar to plant asparaginases. While the $c p h A$ and $c p h B$ genes are frequently clustered, the gene encoding isoaspartyl dipeptidase is located in a different chromosome location.

Some bacteria are able to use cyanophycin as a carbon source for growth in a process that involves extracellular cyanophycinases. The enzyme from Pseudomonas anguilliseptica (CphE) has been purified and shows high specificity for cyanophycin to produce also a $\beta$-Asp-Arg dipeptide, although it exhibits only ca. $27 \%$ identity to the intracellular cyanobacterial cyanophycinase. 


\section{Regulation of cyanophycin accumulation}

In both the unicellular cyanobacterium Synechocystis sp. PCC 6803 and the filamentous heterocyst-forming cyanobacterium Anabaena sp. PCC 7120, the $P_{\|}$protein factor (GlnB) influences cyanophycin accumulation. In the former case, the effect has been shown to involve activation of $\mathrm{N}$-acetylglutamate kinase (NAGK) -a key enzyme of the arginine biosynthesis pathway- by unphosphorylated $\mathrm{P}_{\|}$, which leads to increased production of arginine under conditions of low C-to-N ratio.

In several strains of Anabaena grown under diazotrophic conditions, cyanophycin synthetase and cyanophycinase activities are higher in heterocysts than in vegetative cells. In Anabaena sp. PCC 7120, the transcriptional regulator NtcA has been shown to orchestrate this spatial regulation from a complex promoter arrangement. In contrast, aspartyl dipeptidase levels are higher in vegetative cells than in heterocysts. Accordingly, cyanophycin synthesized at the expense of fixed $\mathrm{N}$ in the heterocyst is transiently accumulated in polar granules and hydrolyzed to $\beta$-Asp-Arg dipeptide, which is transferred to the vegetative cells to be further hydrolyzed to Asp and Arg to be used for metabolism. Thus, the dipeptide represents a nitrogen vehicle in the diazotrophic filament.

\section{Nitrogen control}

In many bacteria, a hierarchy in the order of assimilation of nitrogen sources is established when more than one is available. In many cases ammonium represents the preferred nutrient, given that its assimilation implies a lower energetic cost than that of nutrients that have to be intracellularly transformed into ammonium. Indeed, in most bacteria ammonium exerts a negative effect on the expression of genes encoding elements of the pathways for assimilation of other nitrogen sources, a global regulatory circuit that is referred to as nitrogen control ( $\mathrm{N}$ 
control). At the molecular level, both the array of protein factors (transporters, enzymes, pathway-specific regulators) that are subjected to $\mathrm{N}$ control, as well as the mechanism of ammonium-promoted down-regulation, vary among different bacteria. However, several basic schemes can be recognized, as described below. A key issue regarding $\mathrm{N}$-control circuits deals with signaling of the $\mathrm{N}$ status of the cell to influence the activity of the $\mathrm{N}$-control regulators. In general, high levels of 2-oxoglutarate which, as mentioned above, is a substrate for the incorporation of ammonium into glutamate and glutamine, are taken as indicators of $\mathrm{N}$ deficiency, whereas additionally high levels of glutamine may be sensed as an indicator of $\mathrm{N}$ sufficiency. Whether both indicators or just 2-oxoglutarate are used depends on the metabolic characteristics of the different bacteria.

\section{The $E$. coli cascade system}

The genetic and biochemical mechanisms of nitrogen control have been studied in detail in the E. coli model system, but some of its general characteristics are conserved in the Proteobacteria. In addition, the $\mathrm{N}$-control system shares sensors and signal transduction mechanisms with those regulating the activity of the principal first enzyme for ammonium incorporation into $\mathrm{C}$ skeletons, glutamine synthetase (see section on Regulation of ammonium assimilation).

In E. coli, the gInA gene encoding GS is organized in an operon together with the genes for a N-responsive, two-component regulatory system, the Ntr system (Fig. 10). The sensor/transmitter component, NtrB, is a protein kinase/phosphatase that can specifically phosphorylate or dephosphorylate the receiver/regulator component, NtrC. When phosphorylated, NtrC acts as a transcriptional activator of the main gInA promoter, while dephosphorylation inactivates it. NtrB is able to autophosphorylate at the expense of ATP, and to transfer this phosphoryl group to NtrC, therefore activating it in the absence of any other component. However, the NtrB activities are modulated by its interaction with the $\mathrm{P}_{\|}$factor GlnB. 
$P_{\|}$proteins are organized as trimers, and each subunit can bind one molecule of 2oxoglutarate. Under $\mathrm{N}$-excess most of the 2-oxoglutarate binding sites in $\mathrm{P}_{\|}$proteins will be empty, with the reverse being true under N-deficiency. In the case of $\mathrm{G} \ln \mathrm{B}$, as a result of a cellular situation of $\mathrm{N}$ excess, many of the trimers will contain a single molecule of 2oxoglutarate. This form of G $\ln B$ will bind to NtrB, with two consequences: i) inhibition of NtrB autophosphorylation; and ii) stimulation of the NtrB-mediated NtrC-P dephosporylation. As a result, the levels of NtrC-P will drop and transcriptional activation of the main gInA promoter will stop. As it turns out, a large number of $\mathrm{N}$ assimilation genes are also transcribed from NtrCdependent promoters, which makes NtrBC a global regulatory system. As with NifA (see section on Nitrogen fixation regulation), NtrC-P binds to upstream activator sequences of $\sigma^{54}$ dependent promoters, thus ensuring that no transcriptional leakage occurs in the absence of the activator.

The GlnB $>$ NtrB $>$ NtrC > target promoter cascade is further controlled at the level of the GlnB sensor. In addition to binding 2-oxoglutarate, each monomer in the trimer can be covalently modified by uridylylation through the activity of the $G \ln D$ protein. $G \ln D$ is a bifunctional uridylyltransferase/uridylyl-removing enzyme (UTase) that can apparently sense the cellular levels of glutamine. High glutamine levels determine deuridylylation of GlnB, while complete uridylylation is observed at low glutamine levels. Since binding of 2-oxoglutarate -and probably $A D P$ - to $G \ln B$ is required for uridylylation, $G \ln B$ appears to be a sensor where different signals (glutamine, 2-oxoglutarate, energy charge) can be integrated.

A further level of complexity arises from the existence of another $P_{\|}$protein, GlnK, a GlnB paralog. While GlnB is always present in the cell, $g \ln K$ transcription is under $\mathrm{N}$ control and GInK is present only under N-deficiency. Both proteins can bind 2-oxoglutarate, can interact with NtrB, and be a substrate of UTase, while only GlnK regulates the AmtB channel activity (see section AmtB regulation, above). 


\section{The NtcA circuit of cyanobacteria}

The NtcA regulator was identified as the product of the gene inactivated in cyanobacterial mutants unable to use inorganic nitrogen sources (nitrate, nitrite or $\mathrm{N}_{2}$ ) other than ammonium, and it turned out to have a universal distribution in the cyanobacterial phylum. NtcA belongs to the family of transcriptional regulators that also includes CAP (catabolite activator or CAMP receptor protein -CRP) from E. coli. As other members of this family, NtcA bears in its C-terminal part a helix-turn-helix motif for binding to DNA at sites with a palyndromic structure, which in the case of NtcA includes the sequence signature GTAn ${ }_{8}$ TAC. NtcA can act as a transcriptional activator or repressor depending on the location of the NtcA-binding site in the regulated promoter, and in the most common NtcA-activated promoters, the binding site is separated by ca. 22 nucleotides from a -10 promoter determinant (consensus: $\operatorname{TAn}_{3} \mathrm{~T}$ ). This promoter structure is similar to that of Class II bacterial-activated promoters, in which the binding site of the regulator substitutes for the -35 determinant that is found in constitutive promoters. Besides this, in some other NtcA-activated promoters, the NtcA-binding site is located further upstream from the -10 determinant (Class I activated promoters) or present degenerate NtcAbinding sites requiring the participation of NtcA co-activators. Worth mentioning is the principal role of NtcA in determining the spatiotemporal specificity of activation of genes involved in heterocyst differentiation by acting on different types of NtcA-dependent promoters included in complex promoter regions, as mentioned above.

Because cyanobacteria lack 2-oxoglutarate dehydrogenase, 2-oxoglutarate is mainly used through the GS/GOGAT cycle (see section on Ammonium incorporation into C skeletons). Thus, 2-oxoglutarate levels depend not only on $\mathrm{CO}_{2}$ assimilation through the Calvin/Benson cycle but also on ammonium assimilation through the GS/GOGAT cycle. As such, 2-oxoglutarate levels, which are low in the presence of ammonium, are a good indicator of the C-to- $\mathrm{N}$ balance of the cell. Indeed, 2-oxoglutarate has been shown to bind to NtcA, to stimulate NtcA-binding 
to DNA in regulated promoters, and to be required at the step of promoter melting to establish the open promoter complex for transcription initiation in Class II promoters.

Regarding NtcA targets, a plethora of genes of different $\mathrm{N}$-assimilation pathways and heterocyst differentiation have been individually characterized as regulated by NtcA. Besides that, a global study by chromatin immunoprecipitation has identified more than 2,000 NtcA targets in the genome of Anabaena sp. PCC 7120, including promoter regions of genes involved in $\mathrm{N}$ assimilation but also in other cellular functions such as photosynthesis, central metabolism or DNA replication and repair. This exceptional abundance of DNA binding sites suggests that besides being a global trancriptional regulator, NtcA could serve a structural function as a genome-wide DNA shaper.

\section{Gram-positive bacteria}

The Gram-positive bacteria include organisms for which relevant information on nitrogen control is available. These organisms are B. subtilis and the Actinobacteria Streptomyces coelicolor, Corynebacterium glutamicum and Mycobacterium spp., including the non-pathogenic species M. smegmatis.

\section{Bacillus subtilis}

The soil, sporulating bacterium $B$. subtilis has been intensively investigated as a model of bacterial development and cell biology. In the presence of glucose as a carbon and energy source, the preferred nitrogen source of $B$. subtilis is glutamine followed by arginine and ammonium. B. subtilis expresses two MerR-family transcription factors, TnrA and GlnR, that execute $\mathrm{N}$ control in concert with glutamine synthetase (GInA). MerR-family transcriptional regulators generally contain a helix-turn-helix domain for DNA-binding in their N-terminal region, followed by a coiled-coil domain and a C-terminal effector-binding region that is specific 
to the effector recognized. TnrA and GlnR bind to similar DNA sites of consensus sequence TGTnAnATTTTnTnACA, which results in an overlap of their respective regulons. However, while TnrA acts under nitrogen-limiting conditions, GlnR acts under nitrogen-sufficiency conditions. Under nitrogen deficiency, TnrA is positively auto-regulatory and regulates at least 35 transcriptional units. TnrA activates the expression of operons such as $\operatorname{nrg} A B$ encoding the ammonium-scavenging protein $A m t B$ and its inhibitor GlnK, respectively, ure $A B C$ encoding urease or nasBC and nasDEF encoding nitrate assimilation proteins, and represses operons such as gltAB encoding GOGAT or gInRA encoding the GInR repressor and glutamine synthetase. Under nitrogen sufficiency, glutamine-inhibited glutamine synthetase (GlnA-glutamine) binds to TnrA blocking its DNA-binding activity (and, hence, impeding its regulatory roles) and to GlnR promoting repression of operons such as $u r e A B C$ or $g \ln R A$ itself as well as the $\operatorname{tn} A$ gene. GlnAglutamine-activated GlnR can indeed bind to a wide number of genomic sites, implying that it is part of complex transcriptional regulatory networks. GInR binds DNA as a dimer, and the Cterminal domain of $\mathrm{G} \ln R$ (which differs from that of $\mathrm{Tn} \mathrm{A}$ ) has a role inhibiting $\mathrm{Gln} R$ dimerization and DNA binding; interaction of this domain with GlnA-glutamine releases such inhibition permitting binding to DNA (Fig. 11). This regulation ensures that operons encoding proteins for the assimilation of nitrogen sources alternative to glutamine, such as $u r e A B C$, are expressed under nitrogen deficiency and fully repressed under nitrogen sufficiency. <Fig. 11 near here>

Another layer of $\mathrm{N}$ control in B. subtilis is exerted by GltC, a LysR-family transcriptional regulator that alternatively binds 2-oxoglutarate or glutamate to activate or repress, respectively, the gltAB operon. Activation and repression involves binding to different DNA sites in the intergenic region between the divergent $g / t A B$ operon and $g / t C$ gene. Further, the gltC gene is negatively auto-regulated and also repressed by TnrA, and the GltC protein is inhibited by interaction with the enzyme glutamate dehydrogenase in the presence of glutamate (Fig. 11). This complex regulation ensures maximal expression of $g / t A B$ when 2-oxoglutarate is available and glutamate scarce. 
This brief summary illustrates the complex system utilized by $B$. subtilis to respond to nitrogen availability and the $\mathrm{C}$-to- $\mathrm{N}$ balance. Of special interest is the integration in this system of so-called trigger enzymes, proteins that perform both enzymatic and regulatory functions. Thus, the nitrogen metabolism enzymes glutamate dehydrogenase and glutamine synthetase interact with transcriptional regulators influencing their DNA-binding activities.

\section{Actinobacteria}

The Streptomycetes are the most important antibiotic-producing bacteria. A model strain for this bacterial group in laboratory work is $S$. coelicolor, which can use a wide range of nitrogen sources including ammonium, nitrate, urea, amino acids and some amino sugars. Nitrogen control is executed by $\mathrm{GlnR}$, which is an orphan $\mathrm{OmpR}$-family response regulator unrelated to Bacillus GlnR. The expression of $S$. coelicolor $g \ln R$ is regulated by the availability of nitrogen, as it is induced under nitrogen limitation and repressed under nitrogen excess. The GlnR protein produced under nitrogen limitation acts as a dimer activating or repressing different genes. GlnR frequently binds to two tandem DNA boxes with consensus sequence gTnAc- $n_{6}-G a A A c-n_{6}$. Genes activated by $G \operatorname{lnR}$ include $a m t B$ and $g \ln A$, and genes repressed include ure $A$ and $g d h A$, which may be not necessary under nitrogen limitation in this bacterium. Interestingly, GlnR regulates the expression of many carbon assimilation as well as nitrogen assimilation genes.

C. glutamicum has importance in the biotechnological industry as a producer of amino acids, mostly glutamate and lysine. C. glutamicum shows the capability of assimilating a number of nitrogenous compounds including ammonium, urea or creatinine. Nitrogen control is executed in this organism by the TetR-family transcriptional regulator AmtR that, acting as a dimer, represses at least 33 genes. The AmtR-binding site has the consensus sequence tttCTATn 6 AtAGat/aA. TetR-family proteins frequently respond to small-molecule ligands that function as metabolic sensors. AmtR instead responds to the $P_{\|}$protein GlnK. Under nitrogen deprivation, C. glutamicum GlnK is adenylylated in response to the increase of the cellular levels 
of 2-oxoglutarate. Whereas adenylylation/deadenylylation of $\mathrm{G} \operatorname{lnK}$ is catalyzed by $\mathrm{G} \ln D$, the 2oxoglutarate sensor appears to be GlnK itself. While an AmtR dimer binds to gene promoters repressing gene expression, adenylylated GlnK interacts with AmtR releasing it from DNA and, hence, preventing it from repressing gene expression.

Mycobacterium smegmatis contains both GlnR and AmtR orthologues, of which GlnR appears to be the general $\mathrm{N}$-control protein. $\mathrm{G} \ln \mathrm{R}$ regulates the expression of over 100 genes by binding to about 50 DNA sites with consensus sequence $\mathrm{Gn}_{2} A C n_{6} G n A A C A$, within which $A C n_{9} A C$ appears to be essential. In contrast, AmtR seems to have some specific roles including repression of the urea assimilation operon in competition with activation of the operon by GlnR. Although less studied than in $M$. smegmatis, the response to nitrogen stress in $M$. tuberculosis appears to be mediated also by GlnR.

In conclusion, the Actinobacteria present two interesting variations in the regulation of gene expression in nitrogen control. Streptomyces $G \ln R$ appears to be regulated just at the level of its own expression, and Corynebacterium AmtR is regulated by interaction with a universal Nto-C balance (2-oxoglutarate) sensor, GlnK.

\section{Concluding remarks}

As with so many other metabolic processes, bacteria display a bewildering diversity in their nitrogen assimilation capabilities, and in the way they regulate them. Bacteria, as a group, are able to effectively assimilate most mineral and organic nitrogen sources, from the simplest, exemplified by dinitrogen, which is among the most inert $\mathrm{N}$ compounds and that only bacteria

(and archaea) can attack, to complex, man-made xenobiotics, exemplified by atrazine. Many of these capabilities are often found within the same cell under strict regulatory controls governed by economy considerations: $\mathrm{N}$ assimilation can be quite demanding energetically. As a result, when given a choice, bacteria prefer the least expensive $\mathrm{N}$ source, often ammonium, and organize the regulation of the activity and expression of $\mathrm{N}$ assimilation pathways accordingly. 
Inorganic and most organic $\mathrm{N}$ sources eventually yield intracellular ammonium that is incorporated into $\mathrm{C}$ skeletons as glutamate and glutamine. The $\mathrm{N}$ status of the cell is usually sensed in terms of concentration of 2-oxoglutarate, or the ratio 2-oxoglutarate/glutamine. These signals are transmitted to a cascade of effectors that finely control both the activity of the key ammonium assimilatory enzymes (notably GS and the ammonium transporter) and their expression and that of alternative $\mathrm{N}$ assimilation pathways, often in hierarchical terms.

\section{Acknowledgements}

Work in the authors' laboratory was supported by grants BFU2016-77097-P (AH) and BFU201456757-P (EF) from Agencia Estatal de Investigación, Spain, co-financed by the European Regional Development Fund (EU)."

\section{Further reading}

Amon, J., Titgemeyer, F. and Burkovski, A. (2010). Common patterns - unique features: nitrogen metabolism and regulation in Gram-positive bacteria. FEMS Microbiology Reviews 34, 588-605.

Bender, R. A. (2012). Regulation of the histidine utilization (hut) system in bacteria. Microbiology and Molecular Biology Reviews 76, 565-84.

Boyd, E. S. and Peters, J. W. (2013). New insights into the evolutionary history of biological nitrogen fixation. Frontiers in Microbiology 4, 201.

De Bruijn, F. J. (ed.) (2015). Biological nitrogen fixation. (2 vols.). Oxford: Wiley-Blackwell.

Fong, R. N., Kim, K. S., Yoshihara, C., Inwood, W. B. and Kustu, S. (2007). The W148L substitution in the Escherichia coli ammonium channel AmtB increases flux and indicates that the substrate is an ion. Proceedings of the National Academy of Sciences USA 104, 1870618711.

Govantes, F., García-González, V., Porrúa, O., Platero, A. I., Jiménez-Fernández, A., et al. (2010) Regulation of the atrazine-degradative genes in Pseudomonas sp. strain ADP. FEMS Microbiology Letters 310, 1-8.

Gunka, K. and Commichau, F. M. (2012). Control of glutamate homeostasis in Bacillus subtilis: a complex interplay between ammonium assimilation, glutamate biosynthesis and degradation. Molecular Microbiology 85, 213-24. 
Herrero, A. and Burnat, M. (2014). Cyanophycin, a cellular nitrogen reserve material In: Flores, E. \& Herrero, A. (eds.) The cell biology of cyanobacteria. pp 211-219. Norfolk, UK: Caister Academic Press.

Herrero, A., Stavans, J. and Flores, E. (2016). The multicellular nature of filamentous heterocystforming cyanobacteria. FEMS Microbiology Reviews fuw029, 40, 831-854.

Luque-Almagro, V. M., Gates, A. J., Moreno-Vivián, C., Ferguson, S. J., Richardson, D. J., et al. (2011). Bacterial nitrate assimilation: gene distribution and regulation. Biochemical Society Transactions 39, part 6.

Merrick, M. (2015). Post-translational modification of PII signal transduction proteins. Frontiers Microbiology 5, 763.

Reitzer, L. (2005). Catabolism of amino acids and related compounds. EcoSal Plus doi:10.1128/ ecosalplus.3.4.7.

Rubio, L. M. and Ludden, P. W. (2008). Biosynthesis of the iron-molybdenum cofactor of nitrogenase. Annual Review of Microbiology 62, 93-111.

Valladares, A., Montesinos, M. L., Herrero, A., Flores, E. (2002). An ABC-type, highaffinity urea permease identified in cyanobacteria. Molecular Microbiology 43, 703-15.

Van Heeswijk, W. C., Westerhoff, H. V., Boogerd, F. C. (2013). Nitrogen assimilation in Escherichia coli: Putting molecular data into a systems perspective. Microbiology and Molecular Biology Reviews 77, 628-95. 


\section{Legends for figures:}

Figure 1. Main paths of nitrogen cycling in Nature. The global processes accounting for the bulk of transformations of nitrogen forms taking place in natural habitats are represented. Except for nitrate/nitrite assimilation, in which plants and algae have an important contribution, and of fungi in ammonification, all others are carried out by bacteria and archaea.

Figure 2. Core and some peripheral nitrogen assimilation pathways in bacteria. The assimilation of ammonium through the GS-GOGAT cycle is at the core of nitrogen assimilation in bacteria. Ammonium can be taken up from the medium or derived from inorganic sources of nitrogen including nitrogen gas (dinitrogen), nitrate and nitrite, as shown in the upper part of the scheme. Nitrogen can be obtained also from organic nitrogen sources including the core amino acids glutamine and glutamate. Other organic nitrogen sources, shown here as examples, include urea, arginine, asparagine, aspartate, histidine and the xenobiotics atrazine and cyanuric acid. Amino acids that serve as excellent nitrogen sources can be catabolized producing glutamate and ammonium; atrazine and urea serve as nitrogen sources because they can be catabolized releasing abundant ammonium. The family to which each represented transporter belongs is indicated (for further information on transporter families, see the Transporter Classification Database at http://www.tcdb.org). (Transporters for glutamate ad glutamine are not depicted in the figure.) The names of some enzymes or gene products that participate in the catabolism of the indicated nitrogen sources (amino acids in three letter code) are shown: AnsA, periplasmic asparaginase (B. subtilis); AnsB, aspartase (B. subtilis); AspC, aspartate aminotransferase; Atz, atrazine degrading proteins; GS, glutamine synthetase; GOGAT, glutamate synthase; Hut, histidine degrading proteins; Nar, nitrate reductase; NifHDK, nitrogenase complex; Nir, nitrite reductase; RocF, arginase (B. subtilis), also known as ArcA (Agrobacterium tumefaciens); Ure, urease. The dashed arrow in arginine catabolism denotes two different sub-pathways described in the text within pathway 1 and pathway 2 ([N], nitrogen conserved as glutamate in 1 or as ammonium in 2). Only substrates/products relevant for our discussion are indicated, and stoichiometry of the reactions is not specified.

Figure 3. Structure and scheme of AmtB and its inhibition by GlnK. Structure of the AmtB trimer (A) and the AmtB-GlnK complex (B) from Escherichia coli viewed in the membrane plane. Structure references are 1UTG for AmtB and 2NS1 for AmtB-GlnK. (C) Schematic of the blocking of AmtB by GlnK, which takes place when the ammonium concentration is high, which determines a low $\mathrm{C}$-to- $\mathrm{N}$ balance that results in deuridylylation of GlnK as explained in the text.

Figure 4. Regulation of glutamine synthetase in Escherichia coli. Adenylylation of GS reduces its activity, and each of the twelve subunits can be independently adenylylated/deadenylylated by adenylyl transferase (ATase). High levels of glutamine favor adenylylation, while low levels result in deadenylylation. In addition, ATase activity is modulated by the $P_{\text {II }}$ proteins, GlnB and GlnK. These proteins can bind 2-oxoglutarate (20G) and be modified by uridylylation. Both uridylylation and deuridylylation are catalyzed by UTase. High levels of glutamine favor deuridylylation, while low levels result in uridylylation. Two forms of the $P_{\text {II }}$ proteins are shown. When completely deuridylylated and bound to one 20G, $P_{\text {II }}$ proteins stimulate the adenylyl transferase activity of ATase. When completely uridylylated and bound to three 20G, $\mathrm{P}_{\text {II }}$ proteins stimulate the adenylyl 
removing activity of ATase. GlnK has a much lower effect on ATase, and this is denoted by dashed lines. This cascade system responds, in a highly sensitive manner, to variations in the intracellular $\mathrm{N}$ status, since it senses the levels of both glutamine and its $\mathrm{C}$ precursor (20G). Yellow diamonds, 2-oxoglutarate (20G); red diamonds, UMP; orange diamonds, AMP.

Figure 5. The urea assimilation system. Urea is taken up into the cells with high affinity by an $\mathrm{ABC}$ transporter. These transporters are constituted by a periplasmic substratebinding protein (yellow), two transmembrane proteins (pink) and two nucleotide-binding proteins (green) that hydrolyze ATP in the cytoplasm. Urea is concentrated inside the cell to a level that can be used by the urease complex, which has lower affinity for urea than the transporter. Urease is made up of three different subunits (genes depicted in brown) and bears a nickel-containing active center that is the product of a maturation process carried out by the products of urease-specific maturation genes (genes depicted in blue). All the genes required for urea assimilation are frequently clustered together in bacterial genomes, as exemplified by the urea assimilation gene cluster of the unicellular cyanobacterium Prochlorococcus marinus strain MIT 9113.

Figure 6. Scheme of the electron transfer pathways in cyanobacterial nitrate reductase (NarB) and nitrite reductase (NirA). The cyanobacterial nitrate reduction system is especial in that both reductases use ferredoxin as an electron donor, but at the same time it is illustrative of the nitrate reduction process. Ferredoxin donates electrons (one at a time) to an iron-sulfur cluster in NarB, from which they follow a typical pathway to reduce nitrate to nitrite in a 2-e reduction reaction catalyzed in the Mo-bismolybdopterin-guanine dinucleotide active center. NirA also uses reduced ferredoxin as an electron donor, and here the six electrons required to reduce nitrite to ammonium are transferred (one at a time) through an iron-sulfur center and a siroheme group, in which the reduction of the substrate takes place.

Figure 7. Nitrogenase and nitrogenase genes. A. Nitrogenase structure at $1.9 \AA$ resolution. Two molecules of the dinitrogenase reductase NifH dimer are bound to a central dinitrogenase heterotetramer (2 NifDK). Substrate reduction takes place on FeMo-co (depicted), which is buried within each of the two $\alpha$ (NifD) subunits of dinitrogenase. Also shown is one of the $\mathrm{P}$ clusters that facilitate transfer of electrons from dinitrogenase reductase to the FeMo-co. B. Genetic organization of nif (nitrogen fixation) genes in Azotobacter vinelandii and Paenibacillus sp., examples of nif clusters containing a large number or a minimal set of nif genes, respectively, and in Anabaena variabilis. In the latter, note the split nifD gene, interrupted by an intervening genetic element that is excised upon heterocysts differentiation. Common nif genes are identified: nifHDK, nifEN and nifB. Regulatory genes nifLA are also shown for $A$. vinelandii.

Figure 8. Heterocyst-forming cyanobacteria. (Top) Light micrograph of filaments of Anabaena sp. strain PCC 7120 showing chains of vegetative cells, which perform $\mathrm{CO}_{2}$ fixation through oxygenic photosynthesis and grow, and intercalary heterocysts, terminally differentiated cells that carry out $\mathrm{N}_{2}$ fixation. (Bottom) Transmission electron micrograph of a terminal heterocyst and two adjacent vegetative cells. The intracellular membranes in vegetative cells (thylakoids) are the sites of oxygenic photosynthesis, and the carboxysomes are the sites of $\mathrm{CO}_{2}$ fixation. In the heterocysts, carboxysomes are missing and the intracellular membranes are largely reorganized to form the "honeycomb" membranes, which are sites of active respiration and anoxygenic photosynthesis. The heterocyst has, deposited outside of the outer membrane, a special cell envelope that contains heterocystspecific glycolipids ( $\mathrm{Hgl}$ ) and polysaccharides (Hep). At the heterocyst poles, cyanophycin 
(multi-L-arginyl-poly [L-aspartic acid]) accumulates as a large cell inclusion known as cyanophycin granule polypeptide (CGP) that is also observed by light microscopy (see above).

Figure 9. Regulation of heterocyst differentiation. Nitrogen deprivation elicits an increase in the 2-oxoglutarate cellular levels, and 2-oxoglutarate binds and activates NtcA, which was present at low concentration during growth with combined nitrogen, initiating a loop of mutual and auto-induction of the genes hetR and $n t c A$, which initially takes place in clusters of cells. Concomitantly, the patS gene is expressed in specific cells that have initiated differentiation, and the primary gene product is processed to render the PatS morphogen, which is transferred to neighboring cells inhibiting their differentiation and leading to single cell selection. Structural proteins and secondary regulators are sequentially expressed, with a direct or indirect requirement for NtcA and HetR, that promote differentiation into heterocysts. In the diazotrophic filament of Anabaena, heterocysts, which fix $\mathrm{N}_{2}$, are separated by stretches of ca. ten vegetative cells, which fix $\mathrm{CO}_{2}$. Heterocysts donate organic nitrogen to the vegetative cells, while the vegetative cells transfer organic $\mathrm{C}$ to the heterocysts.

Figure 10. The Escherichia coli cascade system of $\mathbf{N}$ control. Activity of the twocomponent Ntr regulatory proteins, NtrB and NtrC, is regulated by phosphorylation. NtrB can be autophosphorylated at the expense of ATP, and then transfer this phosphoryl group to NtrC (upper central part). NtrC-P can activate transcription through binding to upstream activating sequences (UAS) of N-regulated promoters, in particular the promoter of the $g \ln A-n \operatorname{tr} B C$, but also those of operons whose gene products allow the cell to assimilate alternative nitrogen sources (lower part). The GlnB and GlnK $P_{\text {II }}$ proteins control the levels of NtrC-P. The completely deuridylylated form of the proteins bound to one 2OG (GlnB-20G and GlnK-2OG), found in the cell when levels of glutamine are high (N excess, Fig. 4), bind to $\mathrm{NtrB}$ and stimulate dephosphorylation, and hence deactivation, of $\mathrm{NtrC}$ (upper right part). In addition, GlnB-20G inhibits the autophosphorylation activity of NtrB (upper left part), this ensuring that no further phoshorylation of NtrC-P can take place. Yellow diamonds, 2-oxoglutarate (20G).

Figure 11. Nitrogen control in Bacillus subtilis. (Top) Scheme of the basic actions of the MerR-family transcriptional regulators TnrA and GlnR. Under $\mathrm{N}$ deprivation, TnrA can act as an activator and a repressor of gene expression. Under nitrogen sufficiency (high glutamine cellular levels), a glutamine synthetase (GS)-glutamine complex binds to TnrA blocking its transcriptional activity and to GlnR allowing it to act as a repressor. (Bottom) The LysR-family transcriptional regulator GltC activates expression of the gltAB operon encoding glutamate synthase (GOGAT) under N deficiency (high 2-oxogluatarate [20G] levels). In response to high glutamate levels, $\mathrm{GltC}$ acts instead as a repressor of gltAB and is blocked by binding of the catabolic enzyme glutamate dehydrogenase (GDH). Note the role of GS and GDH as "trigger" enzymes (i.e., they act as both catalytic enzymes and regulators of gene expression). 
Fig. 1

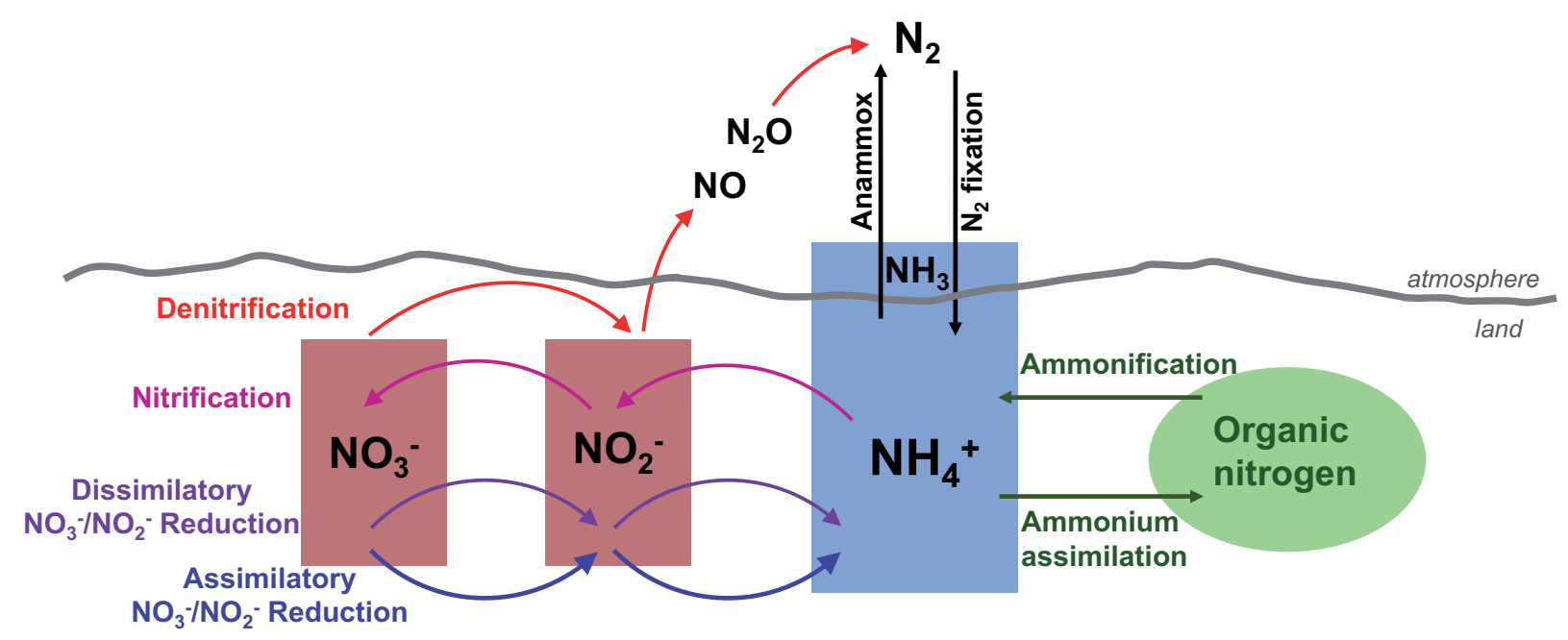


Fig. 2

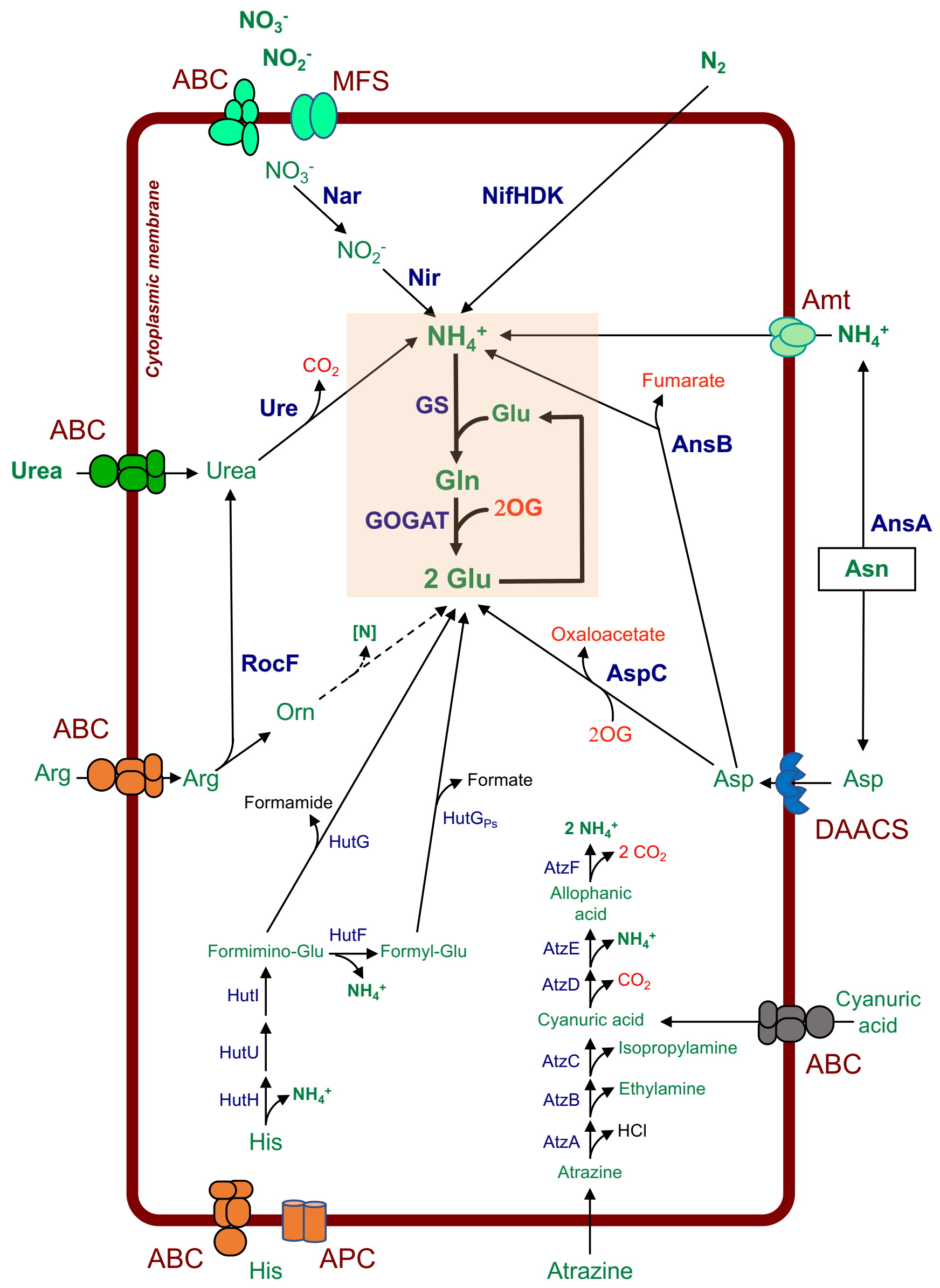


Fig. 3
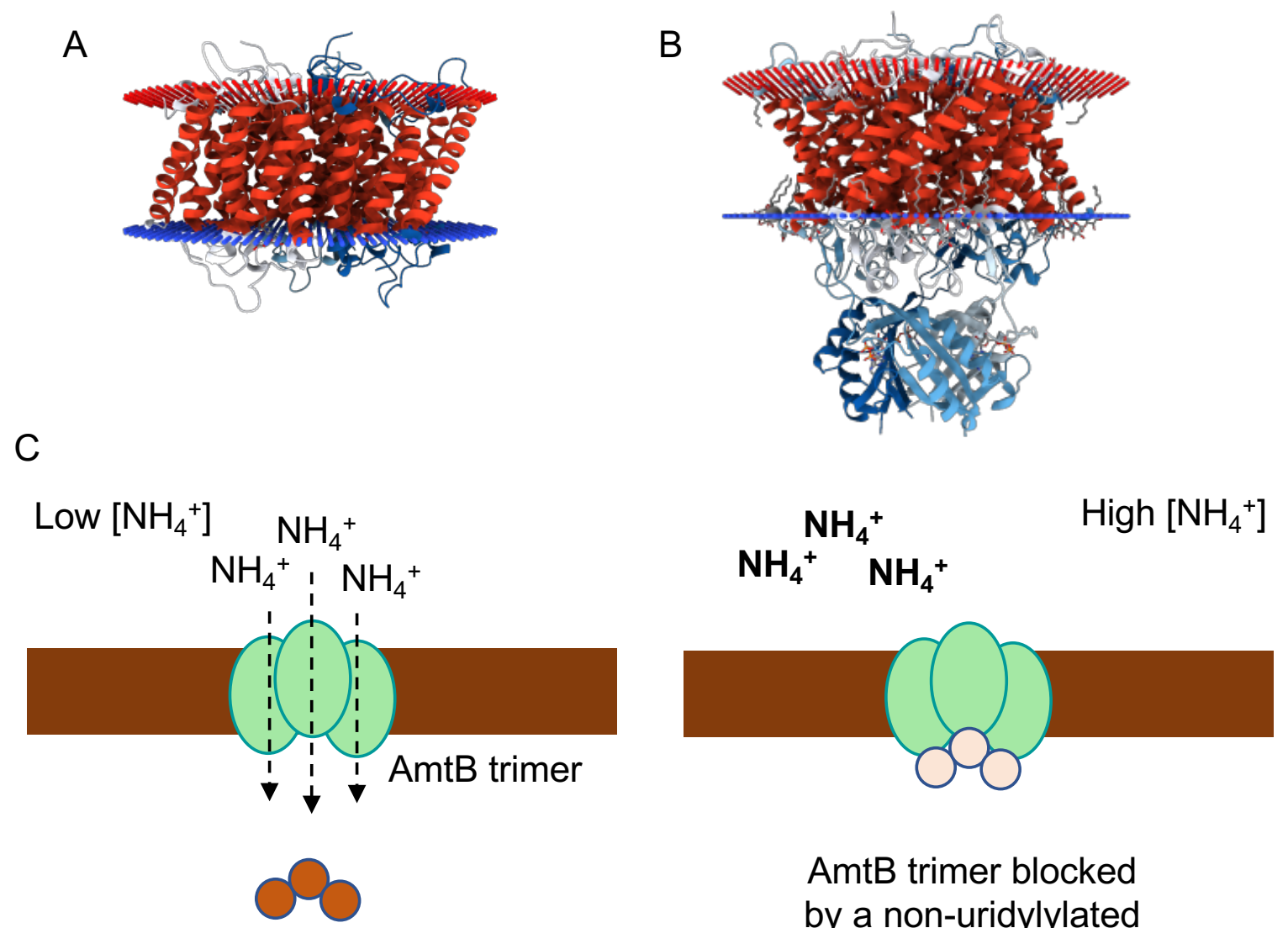

AmtB trimer blocked by a non-uridylylated Uridylylated GInK trimer GlnK trimer 
Fig. 4

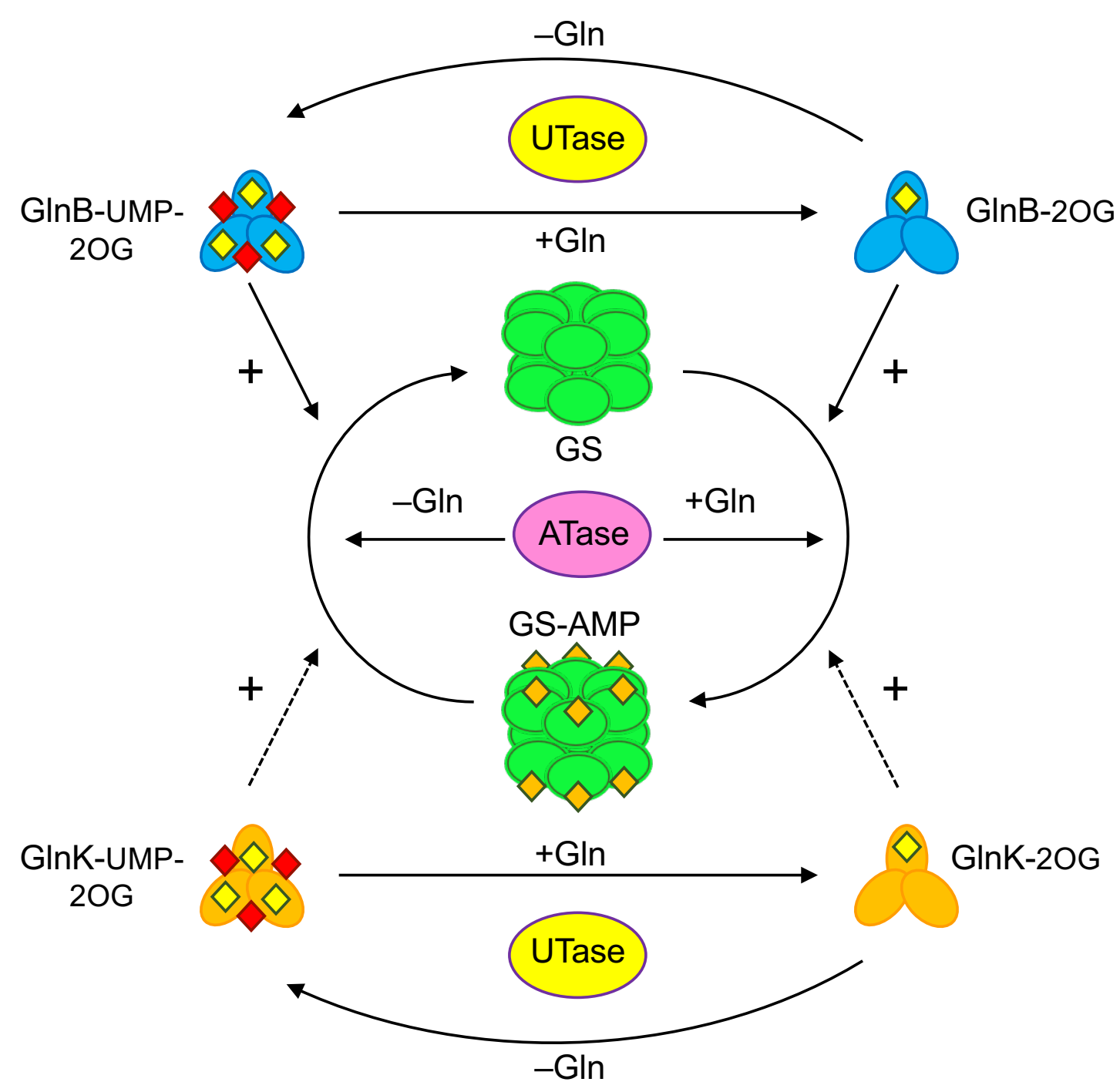


Fig. 5

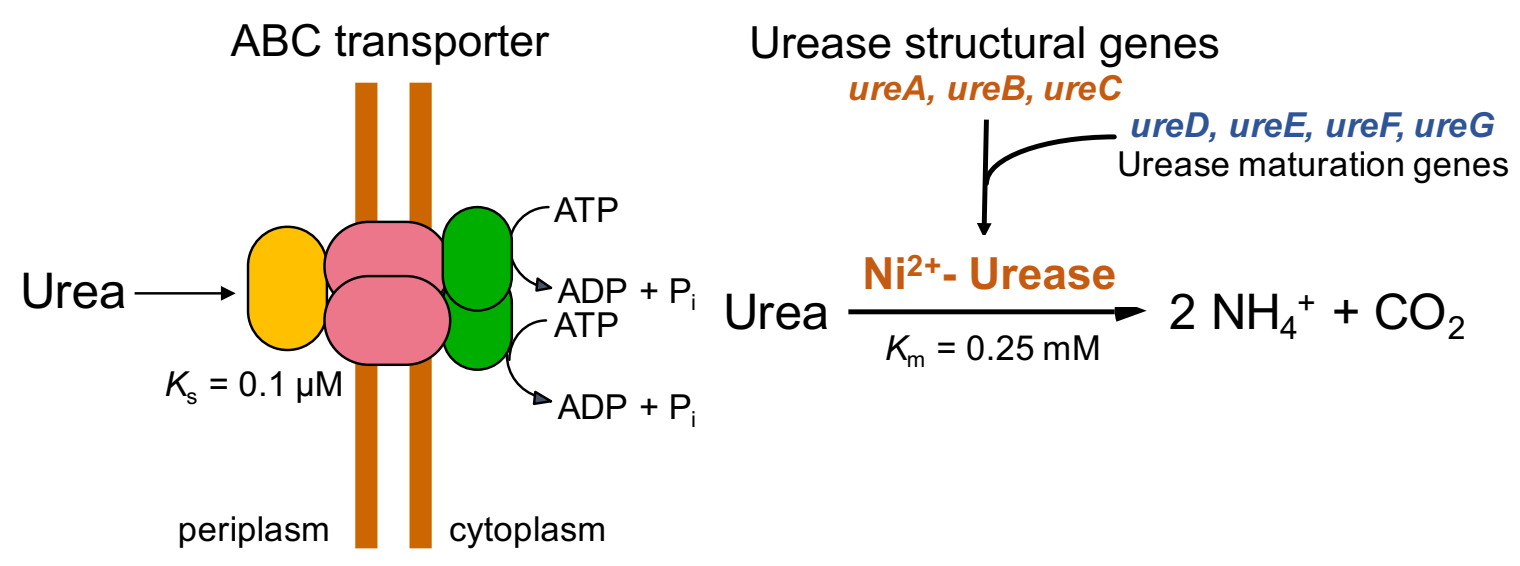

Urea assimilation gene cluster in the cyanobacterium Prochlorococcus marinus MIT 9313

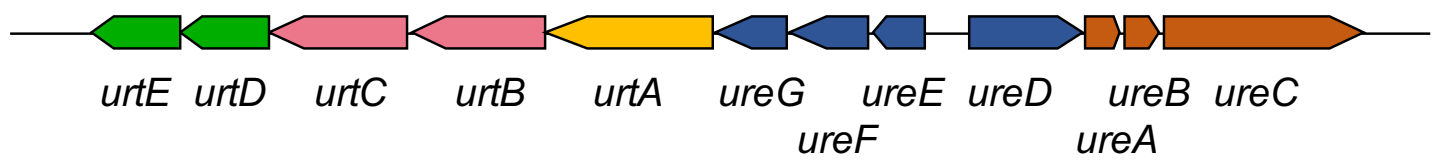


Fig. 6

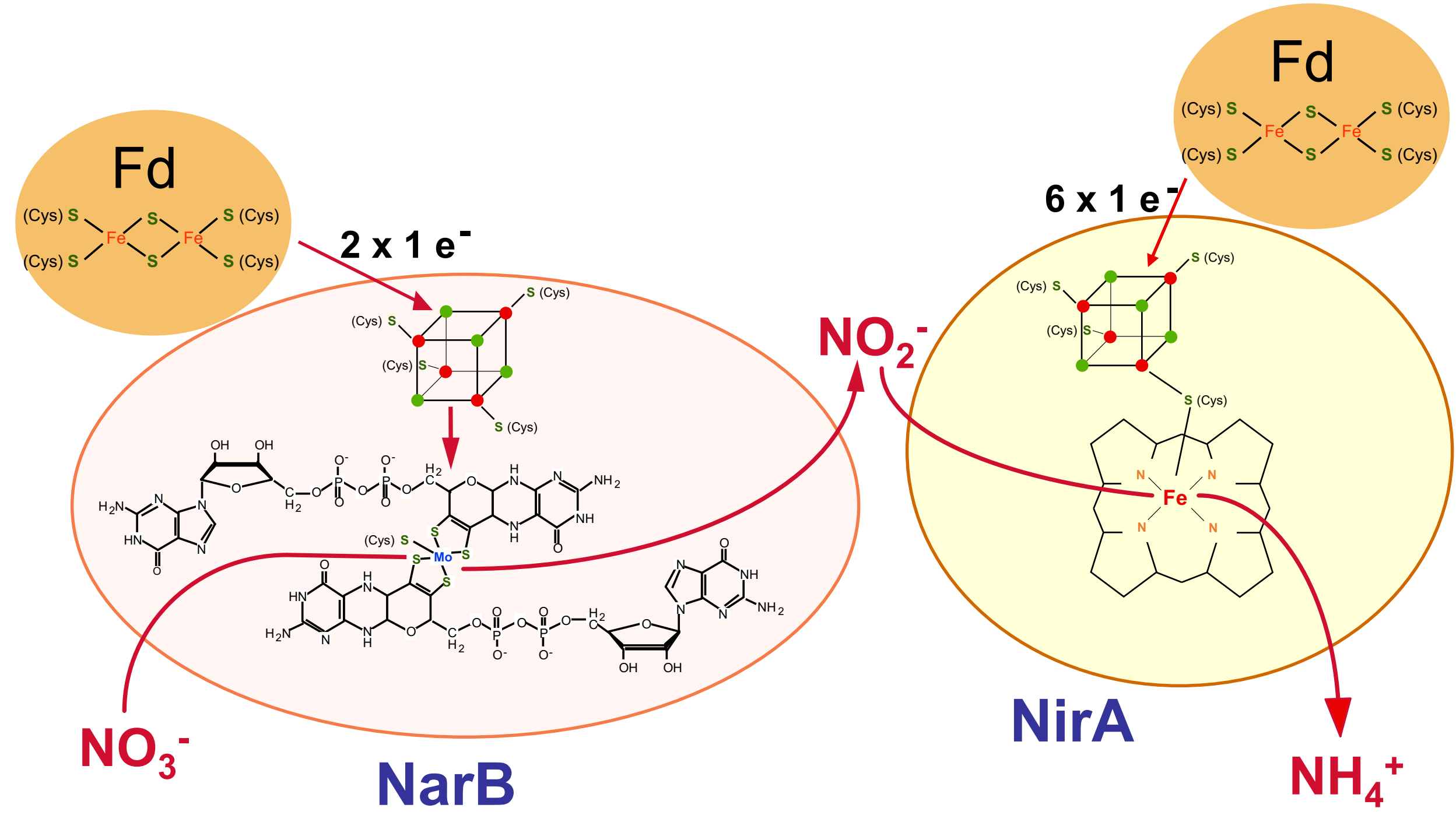


Fig. 7

A

NifDK

$\mathrm{NifH}$

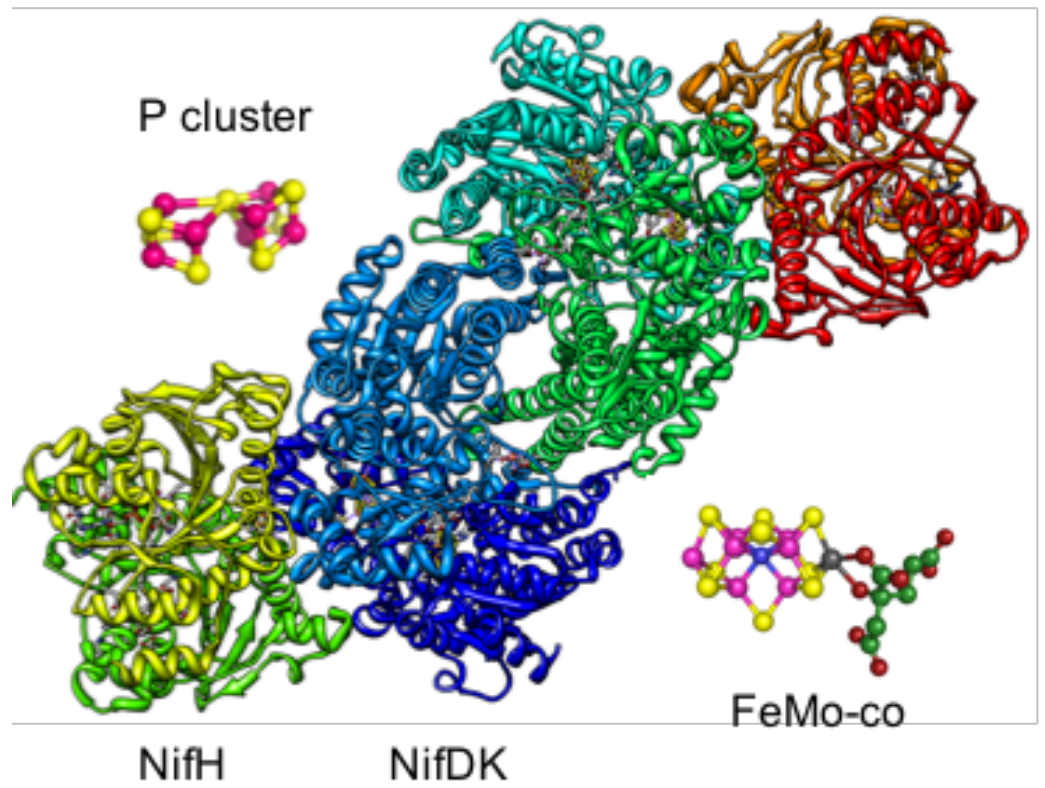

B

Azotobacter vinelandii

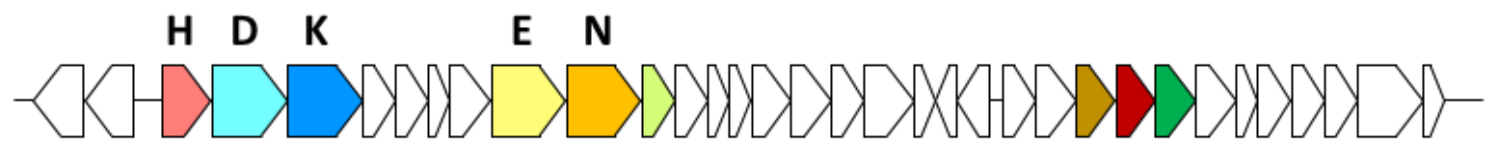

L A B

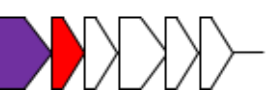

Paenibacillus sp.

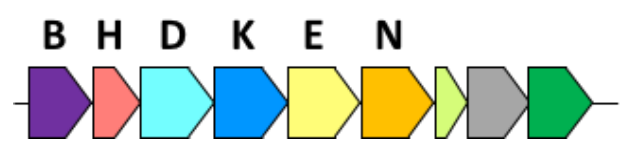

Anabaena variabilis

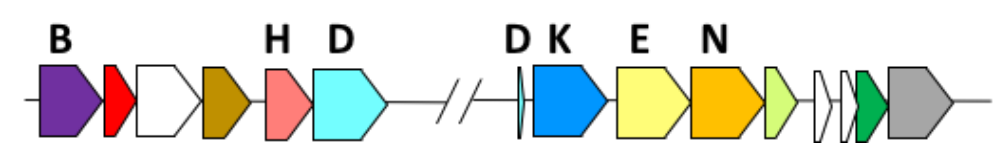


Fig. 8
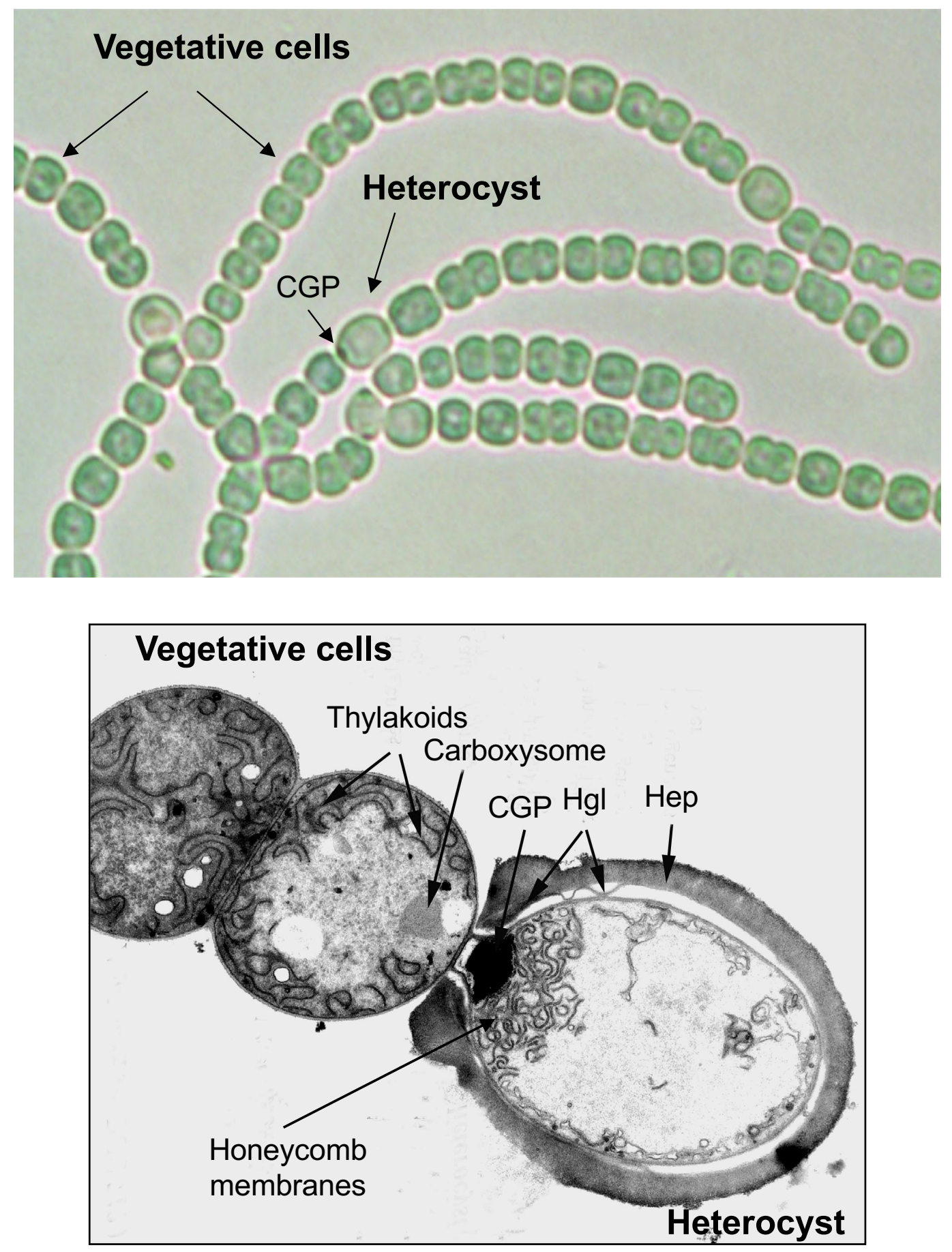
Fig. 9

Growth with combined nitrogen

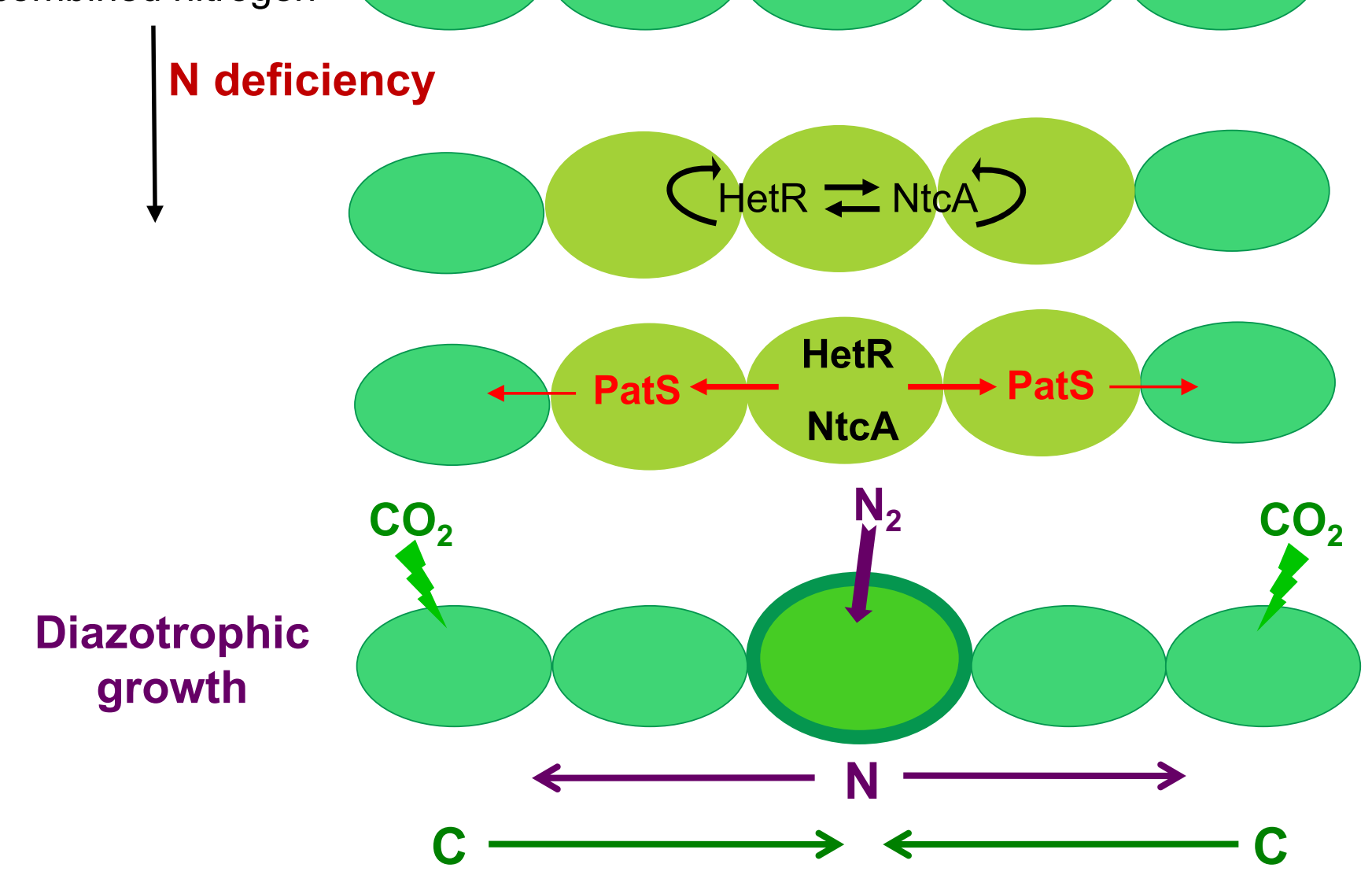


Fig. 10

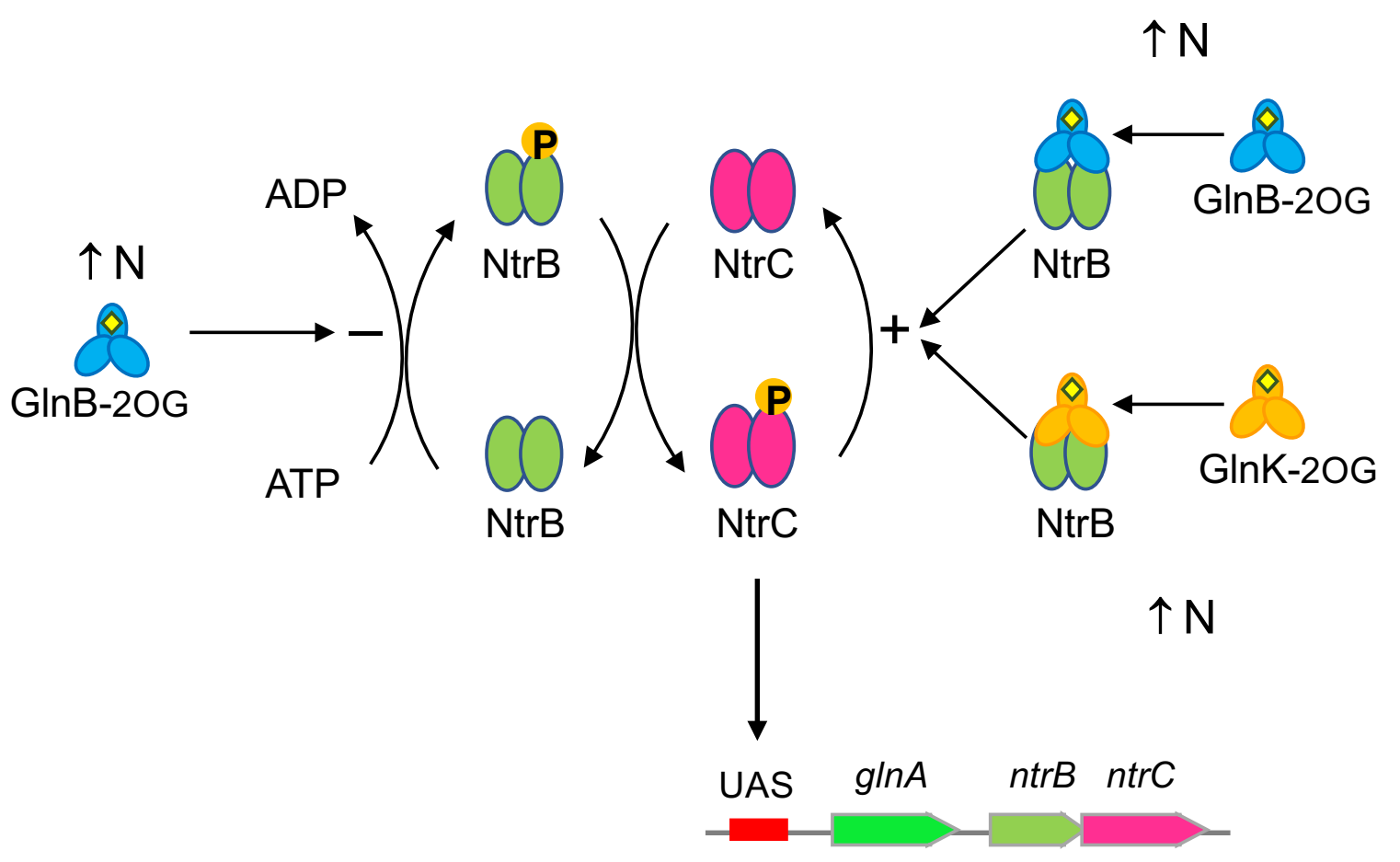

UAS Other $\mathrm{N}$ assimilation systems 
Fig. 11

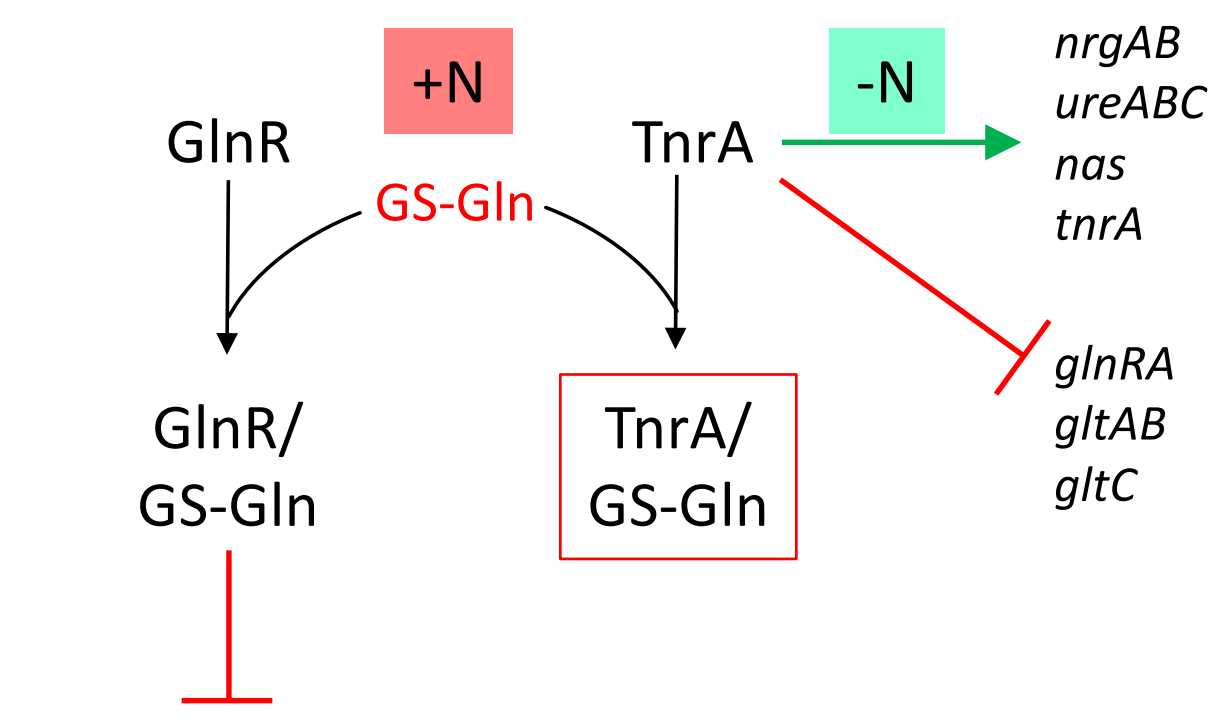

$g \ln R A$, ure $A B C, \operatorname{tn} r A$

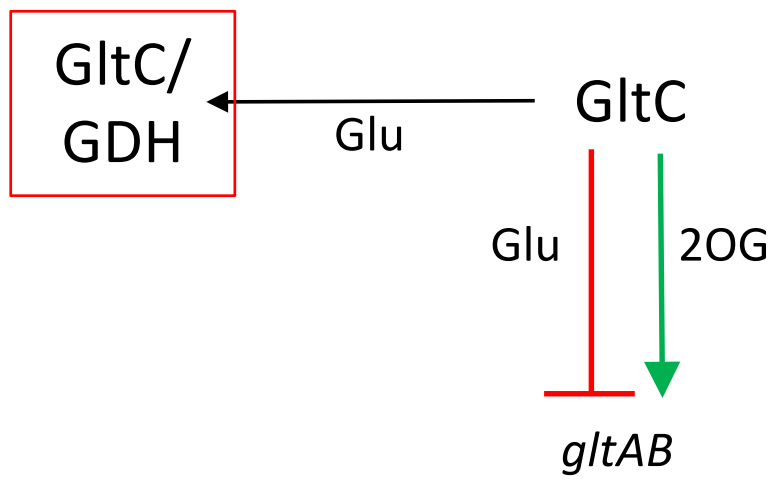

